University of Rhode Island

DigitalCommons@URI

Open Access Master's Theses

2014

\title{
AURAL (RE)POSITIONING AND THE AESTHETICS OF REALISM IN FIRST-PERSON SHOOTER GAMES
}

Ryan P. LaLiberty

University of Rhode Island, rlaliberty@my.uri.edu

Follow this and additional works at: https://digitalcommons.uri.edu/theses

\section{Recommended Citation}

LaLiberty, Ryan P., "AURAL (RE)POSITIONING AND THE AESTHETICS OF REALISM IN FIRST-PERSON SHOOTER GAMES" (2014). Open Access Master's Theses. Paper 301.

https://digitalcommons.uri.edu/theses/301

This Thesis is brought to you for free and open access by DigitalCommons@URI. It has been accepted for inclusion in Open Access Master's Theses by an authorized administrator of DigitalCommons@URI. For more information, please contact digitalcommons@etal.uri.edu. 
AURAL (RE)POSITIONING AND THE AESTHETICS OF

REALISM IN FIRST-PERSON SHOOTER GAMES

BY

RYAN P. LALIBERTY

A THESIS SUBMITTED IN PARTIAL FULFILLMENT OF THE

REQUIREMENTS FOR THE DEGREE OF

MASTER OF ARTS

IN

COMMUNICATION STUDIES

UNIVERSITY OF RHODE ISLAND

2014 


\title{
MASTER OF ARTS THESIS \\ $\mathrm{OF}$ \\ RYAN P. LALIBERTY
}

\section{APPROVED:}

Thesis Committee:

Major Professor Ian Reyes

\author{
Norbert Mundorf \\ Valerie Karno \\ Carolyn Betensky \\ Nasser H. Zawia \\ DEAN OF THE GRADUATE SCHOOL
}

UNIVERSITY OF RHODE ISLAND

2014 


\begin{abstract}
The following study is concerned with how immersive experiences are constructed in first-person shooter (FPS) video games through the implementation of "realistic" audio. Bringing together the three fields of video game studies, sound studies, and science and technology studies in its theoretical framework, this study approaches FPS games as commodities, marketed for their capabilities towards providing the player with an immersive and realistic experience, and constructed in particular ways, for particular ends. The first part of this study explores the context of FPS game audio development, from the earliest days of video games in the midtwentieth century, to the current day. It is argued that the history of FPS games is tightly coupled with the innovation of particular audio reproduction technologies, with the greater history of representation across forms of media, and on a trajectory towards increased immersive realism. The aesthetics of realism as presented in war cinema are taken as a fundamental influence for how immersive and realistic auditory experiences are constructed for contemporary FPS players. The second part of this study takes four FPS games and formally describes them in terms of how the player is positioned as a subject via the game and platform's audio affordances and disaffordances. Finally, both sections are brought to bear on one another in a diachronic account of how subjects have been (re)positioned via game audio throughout the history of the FPS. Merging these threads, ultimately this study argues that the player-subject of FPS games has been aurally (re)positioned on a trajectory shared with the refinement of audio reproduction technologies towards greater immersive realism. Technological development and the aesthetics of realism, as well as the evolution of FPS games into
\end{abstract}


competitive multiplayer formats, have been mutually influential in this, one necessitating the other in a constant cycle of refinement and occasional decline. 


\section{ACKNOWLEDGMENTS}

I would first like to thank Dr. Ian Reyes. Your critical feedback brought this project to level that I did not foresee. While the sheer quantity of emails I sent to you should likely have triggered your email client's "spam" filter, you responded thoughtfully, but moreover, helpfully to each one. I encountered countless walls and mazes during this project, and you helped me to push through those possible, to rethink those I was imagining, and to turn away from those unsolvable. But most of all, you kept pushing me to not give up on this project and to create better work.

Dr. Guo-Ming Chen, Dr. Valerie Karno, and Dr. Carolyn Betensky, your courses provided me not only with some of the knowledge required to complete this project, from theory to methodology, but your assignments and our class discussions provided me with excellent spaces to test my ideas, and your feedback helped me to clarify them.

Dr. Rachel DiCioccio, Sandra Baker, and Donna Cerce, your technical guidance made this project possible, and all of its administrative quagmires less murky.

My friends and colleagues, especially Sarah Ferris, Katie McGwin, Ambrose Curtis, and Colin Syfert, thank your for listening to me ramble on incessantly about subject positioning and video games and for keeping me sane during this long process.

Mom and dad, thank you for letting me use the PC when I was five, and for buying me all of those game consoles. You probably never expected it to go this far. My brother, Justin, thank you for showing me how to type "wolf3D.exe" into the MSDOS command prompt before I even knew how to multiply. 


\section{TABLE OF CONTENTS}

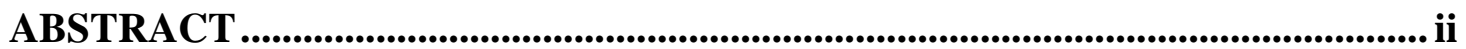

ACKNOWLEDGMENTS .................................................................................................. iv

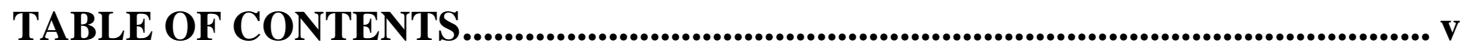

CHAPTER 1 ......................................................................................................................... 1

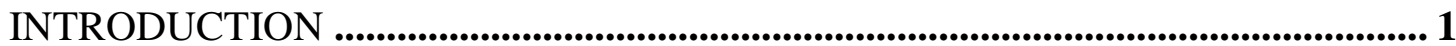

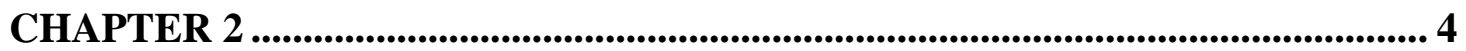

REVIEW OF LITERATURE ......................................................................................................... 4

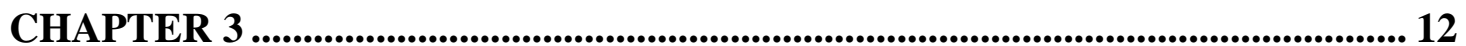

METHODOLOGY ............................................................................................................................ 12

CHAPTER 4 ................................................................................................................................... 21

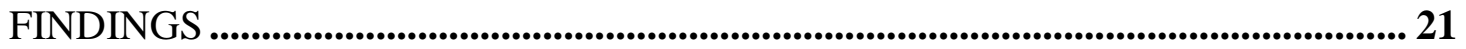

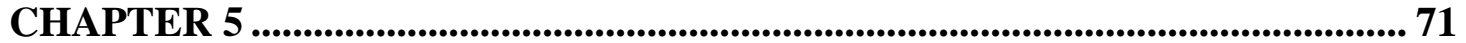

CONCLUSION .......................................................................................................................... 71

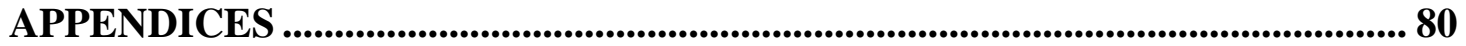

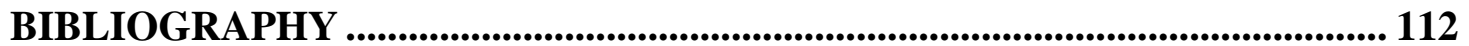




\section{CHAPTER 1}

\section{INTRODUCTION}

Sound design is crucial to the development of any audiovisual medium. It becomes especially relevant when the medium is defined by interactivity, as is the case with video games. Good sound design can make or break a game. It can immerse a player, enveloping them in an interactive, rich soundscape, or distance them through its poor implementation. Of all video game genres, it is in audiovisually realistic games where sound design takes on the largest role. Such games, like military firstperson shooters (FPS), strive to replicate "realistic" soundscapes and emulate our interactions with them, while still featuring musical soundtracks and various other non-diegetic sounds that assist in making video games functionally interactive. Their

goal, as has been the subject in this study, is in constructing immersive gameplay experiences.

While hearing is fundamentally an internal process — sound, after all, may originate out there, but it is only heard in the interaction between brain and ear - the sound object is shaped by exteriors. In the case of the FPS game, these exteriors are physical, i.e., the acoustic environment of the gamer, console hardware affordances, playback technology, as well as cultural, historical, and social from the discursive construction of "realism" to the ways other media have preconditioned contemporary modes of listening. The interactions between the player and game take place in a machine/human network. The relationship between sound design and auditory experience is not a simple linear transfer; it exists in a web of complex relations - 
historical, cultural, and social — between technology and player. Sensory regimes, including those in which the player of a game is positioned as a subject, as this study will demonstrate, do not exist in vacuums. Thus, in FPS video games, sound cannot be studied as just another narrative element, as it traditionally has been. It must be approached at the intersection between culture/history, the body, and technology. Video games are not traditional narratives and they are not films. They must be approached in a way that accounts for what makes them unique, new media - for what makes them games. To do otherwise is reductive; it fails to account for the new possibilities opened up by video games. To understand how sound functions in FPS video games, one must interrogate not only what is heard, but, to use the language of Sterne (2003), why, how, by whom, for whom, and through what means. Answers to these questions will not be sought in player self-report, but rather in the interpretative interrogation of games as texts, augmented by a close reading of the historical, cultural, and social structures from which FPS games and the audio reproduction technologies on which they rely, emerge. Fundamentally, the focus will be on what these games afford sonically in attempting to construct an immersive user experience and how the player-subject is positioned in the auditory regime of the game. The goal is not to construct an ethnographic account of listening practices, but rather explore how the game-text — and the surrounding discourse, from marketing to criticism structures and restructures the possibilities for auditory experience through particular — historically and culturally embedded — affordances and disaffordances. Not only will the positioning of players as subjects be examined diachronically, from the inception of the FPS as a viable genre in the early 1990s to its present day incarnation 
on the Sony Playstation 3, but the very concepts of immersion and realism will be interrogated to evidence not only how immersive experiences are constructed through the implementation of "realistic" game audio, but, moreover, what immersion and realism have come to be defined as, from marketing strategies to a psychological states.

\section{$\underline{\text { Research questions }}$}

My study will utilize the FPS as a case study that I hope will elucidate the complex nodal relations between the hearing body, history, culture, and technology in simulation video games. I stage three research questions. The first two are historical; the third concerns players as subjects of — and subject to - this history.

$R Q_{1}$ : Has the pursuit of immersive realism in FPS gaming led to the emergence of new technologies related to audio reproduction? If so, why, how, by whom, for whom, and through what means have these technologies emerged?

$R Q_{2}$ : As audio reproduction technologies develop, how are they implemented in FPS sound design?

$R Q_{3}$ : How do the changing affordances of FPS games, as brought about through the implementation of new audio reproduction technologies in the sound design process, (re)structure the possibilities for auditory experience? 


\section{CHAPTER 2}

\section{REVIEW OF LITERATURE}

At its core, this study maintains that video games are both a form of play and interactive texts, bridging a long-standing methodological divide that will be detailed in this section. Games themselves - games without the video — are not new and date back to the Paleolithic era. Given this, games as objects of study have been approached in a wide variety of ways, not the least of which are the fields of video game studies, human-computer interaction (HCI), and sound studies, as this section will detail. However, games and those who play them have also been examined from the perspectives of psychology, sociology, and anthropology, ranging from studies of media effects (Ferguson \& Olson, 2014; Willoughby, Adachi, \& Good, 2011) to ethnographic work surrounding particular game-related cultures, whether those be of the video or non-video variety (Christensen, 2006; Rowlands, 2010; Williams, 2006). This study does not wholly discount such work - truly, it has a lot to offer to the comprehensive study of games as they are situated in culture — but this work cannot account for the research questions proposed by this study, questions concerning how subjects are positioned by game texts, how subjects are immersed in the virtual worlds of games, and how these processes are deeply embedded with other technological, social, cultural, and historical processes. This section will proceed by examining some pre-video game studies work, and then weave its way through the emergence of video game studies as a distinct field, its resultant methodological "divide" (and this study's 
position on that divide), and its occularcentrism, concluding finally with a brief review of some video game studies work that has taken into account game audio.

\section{From game studies to video game studies}

According to Aarseth (2001), 2001 brought the birth of video game studies as a distinct field. As he has noted, however, game studies itself is not new. Prior to the advent of the computer, the study of games and play existed in academia. Some earlier seminal works include Huizinga's (1971) Homo Ludens from 1938, which has considered play a central activity in any growing society; Caillois's (2001) Man, Play and Games from 1961, which, like Huizinga, has reconsidered what defines play and how games are introduced into society; and Avedon and Sutton-Smith's (1971) The Study of Games, which, like Huizinga and Callois, has approached games anthropologically, seeking to define "play" and examine its place in society.

Furthermore, the fields of board game studies and sports studies both offer a wealth of insight into the role of games and play in culture. While valuable, however, as Eskelinen (2004) has noted, these insights cannot fully account for contemporary video games as social and cultural practices, while at the same time accounting for what also makes them new media - their digitality.

\section{Video games as narrative, as games, and as hard/software}

The 1990s saw a renewed interest in game studies, concurrent with the growth of the Internet. This was likely due to a newfound interest in "cyberculture" and the newfound possibilities for digital media brought about via the World Wide Web. As Aarseth (2001) has noted, video game studies emerged from older fields, like traditional game studies, complicated by the amplified cultural role of games and their 
new technological remediations. Yet, even prior to the growth of game studies as a field, video game studies takes influence from both literary and film theory in its attention to texts, representation, and their apparatuses of mediation. While the field is not as settled as literary or film studies, as Aarseth (2001) and Eskelinen (2004) have discussed, considerable research has been undertaken that approaches games from a variety of perspectives and via a variety of methodologies

Fundamental to video game studies as it has continued to evolve is the methodological divide between narratology and ludology. Narratology, identified with the work of Murray (1997) and Atkins (2003), among others, approaches games as narratives, albeit in new, technologically mediated form. Scholars approaching video games from this perspective tend to inquire how stories are being told via computing technology. They tend to be interested in the remediation of traditional narrative form and structure and systems of representation as theorized in literary and media criticism. At the perspective's most extreme, video games are simply stories written in code rather than on paper. If narratologists approach video games as extensions of traditional narrative forms, ludologists, on the other hand, taking influence from the work of Huizinga (1971) and Callois (2001), have maintained that games should be understood as formal systems of rules. This approach has been used to describe the work of Aarseth (1997), Eskelinen (2004), Frasca (2003), and Juul (2005). Juul has described the approach succinctly: "A game is a rule-based system with a variable and quantifiable outcome, where different outcomes are assigned different values, the player exerts effort in order to influence the outcome, the player feels emotionally attached to the outcome, and the consequences of the activity are negotiable" (p. 36). 
This system may be presented under the guise of a story, but this story is only a mask upon the underlying abstract mechanics. While this divide has been occasionally situated in mutually exclusive terms, with the distinct narratologist and ludologist camps firmly entrenched on opposing sides of a theoretical battlefield, Frasca (2003), whose work is often identified with the ludological approach, has argued that this debate has been greatly exaggerated and considers the ways in which narratology and ludology can inform one another in the comprehensive study of particular games or of gaming in general as a social and cultural practice. This study has taken a similar position, as will be detailed. First, however, human-computer interaction (HCI) scholarship will be explored, as an approach related to the ludological position.

As Aarseth (2001) has noted, "Games are both object and process; they can't be read as texts or listened to as music, they must be played" (para. 6). To inquire how play occurs can be done at the level of human-computer interaction, the point where games are met by those who play them, at both software and hardware levels. HCI is generally considered to be a subfield of computer science, but its methodology has been applied in various ways to the interrogation of video games as texts. Fundamentally, HCI is interested in interfaces and the ways in which interfaces structure the experience for the user, often with the practical goal of improving user interfaces (UIs). In video games, the interface traditionally consists of the various menus and graphical overlays that dominate the screen and provide information to the user. Additionally, the interface consists of the various mechanisms of controlling game functions, whether those functions be the shifting and rotation of blocks in Tetris (Nintendo, 1989) or the movement in a three-dimensional (3D) space of a virtual 
character. This present study has expanded on these examples of interfaces by taking into account how game sound, and the technologies that produce those sounds, from the sound chip to the speakers, functions as an interface. As Barr (2008) has noted, most HCI research involving video games concerns how they compare to productivity applications, from word processors to CAD software. The aim, more often than not, of such research is to evaluate how well video game interfaces guide the user in order to design better interfaces (Lazzaro \& Keeker, 2004; Malone, 1982; Fulton, 2002;

Pagulayan et al., 2003; Dyck et al., 2007; Fabricatore, Nussbaum, \& Rosas, 2002). While such work is certainly valuable and has its place in video game research, in its focus on evaluation and design, traditional HCI research ignores the wider range of game functions that serve to structure the user experience, from the rules and goals of the game, to the organization of navigable space in the game world, to the pre-existing knowledge of genres and their associated gameplay schemas that serve to guide player positioning vis- à-vis the game. Moreover, HCI research concerning video games is predominantly graphics oriented and ignores game audio. That said, what this study takes from $\mathrm{HCI}$ is its focus on the means of mediation. A video game only exists where the player meets the interface, from the controller to the screen to, this study's focus, technologies of audio reproduction.

Like Frasca (2003), the present study does not deny the importance of the narratological approach to video games, nor does it deny the value of a traditional HCI approach or a ludological approach. Instead, these three perspectives are brought to bear on one another. In answering the research questions at hand, it has taken a ludological and HCI approach to player interaction and game world navigation via 
sound, examining the rules, mechanics, and various interfaces undergirding both . Narratologically, games are taken as texts with particular aesthetic qualities. As a whole however, the present study moves beyond both approaches, situating these games, and their underlying mechanics and representative aesthetics, historically, culturally, and socially.

\section{$\underline{\text { Game studies and occularcentrism }}$}

Regardless of methodological allegiance, the video game has been approached more often than not as a predominantly visual medium. Sound in games has gone understudied, as the graphical aspects of games have received the majority attention by scholars. It has already been noted that traditional HCI research tends to have a graphical bias. While there is a growing body of work that considers techniques of sonification and auditory display in the context of HCI (Herman, Hunt, \& Neuhoff, 2011; Fernstrom, 2005; Walker, 2013; Davison \& Walker, 2008; Ibrahim \& Hunt, 2007), the focus again, like most other HCI work, is on evaluation and design, and moreover, is often not specific to video games. In video game studies specifically, the situation is little different. Kücklich (2003) has maintained that "Usually, the only thing the player knows about the world of the game is what is displayed on the screen" (para. 1). Similarly, Filiciak (2003), in his analysis of massive multiplayer online roleplaying games, has suggested that player-avatar identity is mainly found visually: "Today, the mirror is replaced with the screen" (p. 100). This should come as surprising given that most contemporary video games, but especially audiovisually realistic games like FPSs, are not visual artifacts with some audio elements; they are complete audiovisual works. Yet still, the bulk of analyses of video game play frame 
the player-avatar relationship, and its immersive capacities, in visual terms. Immersion is, more often than not, theorized as occurring through the graphical aspects of a game, rather than concurrently through the sonic. This is not to deny that a body of work concerning game sound exists — it does, albeit small — however, my work represents a new direction.

\section{Game studies and sound}

One can find a wealth of scholarship on game sound if by "sound" the researcher is implying "music" (see: Whalen, 2001; Weske, 2002; Zehnder, 2004; Kaae, 2008; Fritsch, 2013) or "speech," as is the case with most narratological work insofar as it includes narrative elements expressed through dialogue. It is only in the past few years that game studies research has begun to account for the multitude of other sounds that occur within the user experience of a game. Where my work differs from this work on game sound is not in its subject - much of Grimshaw's work has examined audio elements of FPS games apart from music (Grimshaw, 2007a, 2007b; Grimshaw, Charlton, \& Jagger, 2011; Grimshaw \& Schott, 2007). My work is fundamentally different in its approach. I have not sought solely to provide a typology

of audio elements, or abstract these elements and consider how they develop a sense of immersion in the player, as Grimshaw does. These are only part of my mission. On one side, my study could fall under the umbrella of media history pertaining to audio media, relating to work by scholars such as Thompson (2002), Pinch and Trocco (2004), Collins (2008), and Sterne (2003). This, on its own, would not be totally novel for video games. Collins (2008) has already examined the technological history of game audio. However, her work falls more under the umbrella of musicology rather 
than sound studies. Collins's questions, namely how games differ from other audiovisual media in their use of sound and how sound design is limited by the technological affordances of the system, are poignant and important to any study of game audio. Yet, her answers reflect a focus on musical composition and arrangement over other, no less important, audio elements present in FPS video games. My study has accounted more for these other elements, (e.g., environmental sounds, nondiegetic alert sounds, VOIP player-to-player communications, etc.) expanding on Collins's study, at least in its historical elements. Moreover, my study has also accounted for the positioning of the player as subject in and subject to the game, examining how developments of audio reproduction technologies (in the pursuit of immersive realism), and their eventual implementation in video game sound design, result in a (re)positioning of the listening player-subject. While visual positioning has hardly changed, the FPS is, after all, defined by the first-person perspective, aural positioning has been altered dramatically. How exactly this has occurred through the changing affordances of the FPS has been an object of my research. The scholarship, up to this point, has not yet merged these points. 


\section{CHAPTER 3}

\section{METHODOLOGY}

\section{$\underline{\text { Design }}$}

The present section will describe the methodology undertaken in this study. Two linked approaches have been undertaken: (1) a directed historical account of console gaming platforms, and (2) the analysis of four seminal first-person shooter (FPS) games to evidence how subjects have been positioned diachronically in aural game spaces. Both of these are undergirded by theory from the fields of sound studies and science and technology studies. Specifically, the work of Sterne (2003) will be described and related to the present study, as his works provides the theoretical framework on which this study's methodology has been built. The first approach serves to contextualize the second, situating the diachronic examination of aural subject positioning in FPS games in its proper historical, cultural, and social context. $R Q_{1}$ has been investigated via this first approach; $R Q_{2}$ has been largely investigated by the second. Answers to $R Q_{3}$ have been sought at the merger of these two approaches.

\section{Theoretical framework}

In answering my historically informed research questions, I have relied heavily on scholars in the related fields of sound studies and science and technology studies. Pinch and Bijsterveld (2004) have defined sound studies as "an emerging interdisciplinary area that studies the material production and consumption of music, sound, noise, and silence, and how these have changed throughout history and within 
different societies" (p. 636). These areas are not new, but as Pinch and Bijsterveld have noted, the broader perspective of sound studies is. While fields like musicology and the social sciences like sociology and psychology have a lot to offer a study of sound in society or sound in media, their approaches and goals are fragmented, as Truax (2001) has noted at length. Sound studies seeks to unify these goals and approaches under one field. Seminal texts include Ihde's (2007) Listening and Voice, a phenomenological account of aural experience; Schafer's (1977) The Soundscape, which has laid out early theory and methodology for studying auditory landscapes; Attali's (1985) Noise, which has examined the connection between music production and political and economic power; Corbin's (1998) Village Bells, which, through an analysis of the village bell, has attempted to "map" the soundscape of nineteenthcentury France and explores the power of sound to regulate life; Bull's (2000) Sounding Out the City, which has sought to ethnographically describe practices of listening in the contemporary urban environment; and Smith's (2001) Listening to Nineteenth Century America, which has explored the differing soundscapes of the northern and southern United States in the antebellum era, through a variety of primary sources and literary work. This is by no means an exhaustive list, but it represents some of the diverse questions asked and answers offered by those writing in the field.

My theoretical framework has been heavily inspired by the materialist sound studies approach of Sterne (2003), who has studied the history of audio reproduction technologies through a consideration of the sociocultural contexts of their innovation. Reproduction technologies, he has argued, result from particular social relations and 
practices. Bringing in the work of Latour (1993) from the field of science and technology studies, Sterne (2003) has interrogated the "networks and assemblages from which technologies emerge" and the ways such technologies speak of "their connection with human practice, habitat, and habit" (Sterne, 2003, p. 8). "History," as Latour has written, "is no longer the history of people. It becomes the history of natural things as well" (Latour, 1993, p. 82). Technology, as both Latour and Sterne have argued, embodies particular practices and perceptions and these practices and perceptions are situated historically. It, and the context of its production and implementation, can be analyzed to evidence the particular "crystallization" of the "repeatable social, cultural, and material processes" that characterize it (Sterne, 2003, p. 8). Sterne's major element of study, merging the fields of science and technology studies and sound studies, is the transducer, a mechanical element analogously found in the human ear and existent in every audio reproduction technology, from the gramophone to the latest studio reference system. Thus, he has situated the development of the mechanical transducer - the core element of an audio reproduction system, devoted to converting sound waves to electric current or corollary mechanical motion — on a trajectory with increasing medicalization of the human body throughout the nineteenth century. Merging these trajectories, he has argued that "the history of sound reproduction is the history of the transformation of the human body as an object of knowledge and practice" (p. 50-51).The transducer, both the physiological and mechanical element, was thus approached as a cultural artifact. Through this close cultural study, Sterne has sought to elucidate not only the sociocultural practices from which audio reproduction technologies emerge, but also 
how these emergent technologies alter these same (and other) practices and relations. Connecting Sterne's approach to the study of the positioning of listening subjects in FPS video games, one must interrogate not only what is heard, but why, how, by whom, for whom, and through what means. While Sterne has reminded us that hearing is fundamentally interior, shaped by each individual's body, sound is always mediated by exteriors, and not necessarily the most obvious exteriors, like the acoustic properties of a listening space, but culturally bound practices ranging from capitalism to the medicalization of the human body, as in his study of the phonograph (p. 343). Those with an ear lent to audio reproduction technologies, surely of which video games are a large part in this century, exist as both physical and social beings. As Sterne, in his conclusion, has noted, while "our bodies must be able to transform physical vibrations into perceptible sounds," we still "must know how to hear and listen to those sounds" (p. 343). Thus, the soundscape consists of more than just the physical vibrations in the air, but also the objects that create, destroy, and alter those sounds, i.e., that mediate those sounds. Mediation is no longer just the intervention of an obvious technology, like a set of speakers or a television screen. Rather, insofar as a sound is affected (and sound is always affected), it is mediated, whether that mediation result from the acoustics of a space, the shape of the pinnae, the sample rate of a video game console, or the practices through which one has been conditioned to listen. As culture and technology, including audio reproduction technology, are irreducibly intertwined, so must the study of either attend to both. To extend Sterne's method to our present object, video games cannot be approached sonically as just another audiovisual media. They emerge from particular practices and contexts that 
serve to position those who play them in particular ways. This study has accounted for both of these processes.

\section{Principles of method}

As it is hoped this study will elucidate, video games espouse particular sensory regimes and position player-subjects within these regimes. As Sterne (2003) has situated his object culturally and historically, so too have I, by examining the varied practices undergirding the reproduction of audio in FPS games and the promotion of immersive experiences. I have examined material practices at two levels: (1) at the level of platform and game development and (2) at the level of the user experience.

\section{(1) Of the development of the platform and game}

As Montfort and Bogost (2009) have developed at length in their "platform study" approach to the Atari video game console, "Work that is built for a platform is supported and constrained by what the chosen platform can do" (p. 3). Thus, in the process of game development, a game is designed for a particular platform. That platform fundamentally delimits what can be implemented in a game. In exploring, then, how particular games structure the user experience through particular affordances and disaffordances, it must always be taken into account that games are designed for particular systems and every system has precise capabilities that cannot be overruled. At the level of the game, the affordances and disaffordances that are built in to the game in the process of development are the absolute boundaries from which the user cannot stray. However, to return to Sterne (2003), the affordances and disaffordances of platforms are situated in particular contexts. The history of technology is not always the history of forward progress, but occasionally of decline, 
stasis, or total reversal. Thus, in examining how a game platform affords or disaffords particular audio reproduction possibilities — and by extension how a game does the same via its mechanics, developed under the constraints of the platform — not only should these possibilities themselves be studied and the constraints that have produced them (the what) but also the why. Under what conditions were these limitations implemented? What may have been the present motivations behind innovation, stasis, or decline, i.e., the removal of features?

\section{(2) Of the user experience}

A video game and the platform it has been designed for, as an apparatus, positions its subject (the player) in such a way that a desire to adhere to the rules of the game is unconditional and freely accepted. Adherence to rules — and not just the rules of gameplay that the player has the choice to adhere to or not, but the structural and algorithmic rules that are programmed in and unbreakable — is necessary to play the game, and furthermore, to win the game. These various rules, uncontestable, afford particular actions on the part of the player and disafford others. Agency exists to be inscribed within the parameters present, parameters so pervasive that they are accepted unconditionally and without pretense. Rules thus limit and delimit what is possible in the space of a game and it is in this context that the possibilities for play come about. Moreover, it is in this context that the player is positioned as a subject — split off from the outside world via the technology of mediation, the player is reconstituted as a acting subject in the virtual world of the game, as an interactant and not a mere inhabitant. Largely what separates films from video games, apart from their technical aspects of production, dissemination, and reception, is interactivity; realism in cinema 
is passively consumed while realism in video games is constructed actively and in real time via the game apparatus of interaction.

Bogost (2008) has understood such rules as espousing particular "rhetorics." He examines the "rhetoric of video games" through America's Army: Operations (U.S. Army, 2001), a massive multiplayer online (MMO) FPS originally funded by the U.S. government and developed as a combat simulator for the U.S. Army. As Bogost has written: "video games are not just stages that facilitate cultural, social, or political practices; they are also media where cultural values themselves can be representedfor critique, satire, education, or commentary" (p. 119). While digital gaming is based fundamentally on interactivity, this interactivity takes place within a framework of rules. Bogost has employed the term "procedural rhetoric" to discuss the practice of arguing through process and algorithm. In the case of America's Army: Operations, not only is the game mechanically and physically realistic, it is procedurally realistic as well. Players are forced to follow the Army's real-life Rules of Engagement (ROE). Failure to do so will pull a player from the game mission and place them in "jail" temporarily, awaiting a military tribunal. Repeated offenses can lead to being banned from the game. In another example of procedural realism, the game's point system mimics the U.S. Army's actual chain of command, which can be negatively affected by ROE violations and positively affected by completing certain mission objectives, just as in the actual military. Fundamentally then, according to Bogost, America's Army: Operations argues a particular perspective of what combat should be. The rules of the game, the affordances and disaffordances given by the game space, limit transgression. Can we extend this however, in a way that Bogost does not, to consider 
not just the way game design espouses ideology, but the ways in that it prescribes (and necessitates) certain sensory regimes? In this way, the rhetoric of video games can be extended to include the sensory schemata necessary to play the game. By interrogating the game as a cultural artifact, much as Sterne (2003) has done with the transducer, by teasing out the game's mechanics and the codes and practices enmeshed within, we may be able to reveal the sensory regimes assumed and privileged by the game space in which the subject is positioned, in the affordances and disaffordances of the game space.

\section{$\underline{\text { Data collection }}$}

In exploring the material dimensions of platform and game development, the present study has relied on a variety of historical sources, just as Sterne (2003) has done in accounting for the social, cultural, and historical contexts from which technologies of audio reproduction have originated. These sources include existent histories of video games and platforms, specifically, the work of Collins (2008) who, as previously mentioned, considers the historical development of audio reproduction technologies in video game sound design, and Donovan (2010). Beyond secondary source material, this study has taken into account primary source material, from critical reviews to advertisement copy to interviews with game developers in establishing its story of how and why new technologies are implemented in game development.

In exploring the material dimensions of the user experience, four FPS games (Wolfenstein 3D (1993), GoldenEye 007 (1997), Halo: Combat Evolved (2001), and Battlefield 3 (2011)) have been played and formally described with the goal of 
interrogating how the subject is positioned differently via game audio, embedded in different platform affordances, and structured furthermore by the implementation of differing audio reproduction technologies. This work has been contextualized and guided by the historical work examined in exploring the material dimensions of platform and game development. The formal procedures and descriptive work of this textual analysis can be found in Appendices 1 and 2, respectively. How subjects have been (re)positioned diachronically will be detailed in the Findings section of this study, contextualized via the historical, cultural, and social work described above. 


\section{CHAPTER 4}

\section{FINDINGS}

\section{Media history of platforms}

As Juul (n.d.) has noted, the history of video games is "in part, a history of technology" (para. 1). Thus, In order to best understand how the player has been (re)positioned as an aural subject through the history of the first-person shooter (FPS), some in-depth knowledge of the hardware and software that enable the shifting possibilities for sound design is necessary. The focus here will be on the four systems that are linked to the present game sample: the Super Nintendo Entertainment System (SNES), the Nintendo 64 (N64), the Microsoft Xbox, and the Sony Playstation 3 (PS3). The following sections will attempt to situate the analysis of shifting subject positions in the greater historical, cultural, and social context of the very technologies that enable this positioning to occur and change, at the level of the platform. At the level of the game, how this technology is realized in particular design strategies has been explored, insofar as these strategies serve to position and (re)position subjects in the user experience. The present technologies, and the context of their production and implementation, can be analyzed to evidence the particular "crystallizations" of the "repeatable social, cultural, and material processes," to use the language of Sterne (2003), that characterize them (p. 8). It is this paper's contention that the history of video game technology, as concerned with representation and simulation, can be best understood on a trajectory coupled with immersive realism. This present section will 
begin by exploring "realism" in media texts, historical and contemporary, situating the concept of realism discursively. While video game realism has a significant prehistory in film, as will be briefly addressed in this section — and thus the aesthetics of video game realism derives significantly from aesthetic strategies established in these other media - in their interactivity, designers of video games have sought to code realistic experiences in newly mediated ways, that evidence video games as unique new media. While immersive gaming experiences may be constructed in a multitude of ways, from controller design to visual authenticity, this present study is concerned with game audio and the aesthetics of realism. As the coming sections will explore at length, how has what counts for sonic realism been constructed? Relevant texts from the pre-history of game realism in film, to the marketing and industry materials surrounding game texts, to player self-report on forums and in the video game press, to recent scholarship in video game studies, will be explored to evidence this. Finally, this section will examine the intersections between these discursive processes and the history of audio reproduction technologies in video games, all aimed at providing answers to $R Q_{1}$. The following section will examine how subjects have been positioned diachronically in FPS video games via the implementation of the new technologies examined in the previous section, providing answers to $R Q_{2}$ and $R Q_{3}$.

\section{$\underline{\text { Realism }}$}

While a rich analysis of the construction of realism in representational media like drama, radio, and film is outside the scope of this paper, such a study would be illuminating as pre-historical and pre-conditional to the construction of "realistic" gameplay experiences - and to the very essence of what "realism" constitutes. In its 
place, a brief excursion into the aesthetics of realism as they are produced in film sound, specifically the sounds of war cinema, will suffice.

Chion (1994) has written at length about the nature of "reality" in film sound. Ultimately, "realism" is not synonymous with "reality," those experiences of phenomena that are lived. Realism, in film, is constructed in the transposition of reality (three-dimensional) to a two-dimensional (2D) audiovisual format:

$[\ldots]$ the sound heard at the end of the process is the product of a preexisting reality plus conditions of reproduction. This end product is a specific reality: neither the neutral transmission of a sound event, nor an entire fabrication by technical means. (p.103)

Moreover, realism is not just constructed at the intersection of the actual object and the mediation of the recording apparatus — recording after all is never neutral and its product always bares its traces — but realism is designed and created from the ground up, and often so with little true resemblance to reality, such as in the creation of Foley sound effects. In Chion's terms, realism does not emerge from reproduction; rather, realism is the product of rendering. Experience in the real world is guided by all five senses simultaneously. One's experience of morning is not just seeing the sun and hearing the rooster crow, but as Chion has continued, the heat of the sun is felt, the light burns the corneas, and presumably one smells the exhaust of the garbage truck as it passes by on its morning run and one tastes their stale breath. "On screen," Chion has written, "the audiovisual channel has to do all the work of transmitting [this scene]: the filmmaker must 'render' [it] by the sole means of image and sound (p. 113). Where the other senses are shut out — there is no olfactory, gustatory, or realistic tactile feedback available in cinema - the senses of vision and audition are heightened in an attempt to fill the gaps and to render the other senses in a 
purely audiovisual medium. The act of rendering thus has the goal of conveying the feeling or perception that would otherwise be conveyed holistically by the other senses.

Moving to war cinema, where this study locates the greatest influence on FPS aesthetics, an automatic weapon would be deafening if fired indoors to anyone around; yet, many films feature lengthy indoor gun battles with protagonists, antagonists, and bystanders seemingly sonically unphased. The $130 \mathrm{~dB}$ hollow pop of a real handgun is transposed, is rendered, as a roaring, but quiet, firecracker. A furious gunfight paradoxically becomes sonically clear. In the cinema, the gunfight is devoid of the intersecting reverberations and crossing of dynamic thresholds that would muddy the soundscape if heard in lived experience. But who would want to watch a gunfight that ended with all of the participants writhing on the ground with horrific ear pain? For Chion, as these codes are proliferated, as reality is rendered and not reproduced, what is called upon as reality after the credits roll is the rendered sound and not the sound of the thing itself. Reality as experienced in the direct audition of some thing and its emergent acoustic characteristics is overrode by the aesthetic choices of cinema ${ }^{1}$, "the more we use recorded and/or transmitted sound, the more we mythify its contrary" (p. 104). Such is the art of Foley sound, where sounds referential to on-screen elements are either created from the ground up or augmented in post-production from the recorded and edited sounds of frequently unrelated props and field recordings. To the viewer and the auditor, these effects convey a reality more real than real.

\footnotetext{
${ }^{1}$ Chion's (1994) understanding of mediation differs from that of Sterne (2003). While Sterne (2003) has situated the act of listening as that which is mediated, for Chion (1994) it is the sound itself that is mediated.
} 
As Wolf (1997) has noted, bridging the gap between cinematic realism and game realism, standards of media realism in video games have been set by film and television (p. 12). Fundamentally, this study argues that the aesthetics of cinematic realism are a precursor to the aesthetics of realism in FPS video games. The majority of players of FPS games are not combat veterans and have no lived experience of warfare or its associated sounds of which the audio in FPS game would seem to take as its referents. Given this, players must still be able to understand the action and events of the game, and how to interact in war scenarios. Thus, these games are designed utilizing the aesthetics of cinematic warfare; while most players are not combat veterans, most are familiar with war cinema. As this study demonstrates, the codes of realism in war cinema do not end at narrative arcs and particular character types, but include as well the representational elements of warfare, both sonic and visual, from the sight of gunshot wounds and muzzle flash, to the sounds of automatic weapons and their acoustic characteristics when fired in certain spaces. According to Gieselmann (2007), FPS video games borrow heavily, in terms of narrative, aesthetics, and the design of mechanics, from war cinema. For example, he has made the case that Doom (id Software, 1993) found influence in First Blood (Kotcheff, 1982), in its proffering of a "dumb muscle machine that was trained by the military to kill everything that moves" as the protagonist. Neither portray an authentic depiction of war, yet as the majority of viewers and players have no knowledge of what war actually is like, such works come to stand in for war, as referents. FPS video games, then, do not attempt to reproduce reality, as the reality of warfare is foreign to the majority of the genre's players, but to remediate reality as it is expressed in cinema - 
what could be called realism. How then can realism be understood in the context of video games, and specifically, audiovisually realistic games like the FPS shooter? Realism, as a concept and a design goal, has been constructed in marketing, in game design instruction, in supplemental game materials, at industry events, and by users and has been discussed at length theoretically in much of the scholarship surrounding video games. Unfortunately a full archival study aimed at interrogating how realism has been constructed discursively in the practice of video game development, marketing, and play is outside the scope of this project; it is my hope that a few examples, coupled with the subsequent theoretical discussion, will suffice.

\section{The construction of realism in video game discourse}

There is little denying that many video games have been marketed and judged based on their realism and immersive capabilities, regardless of the channels through which realism is constructed. For audiovisually realistic games, like the FPS, this is especially so. Even Wolfenstein $3 D$ (Wolf $3 D$ ), considered by many to be the first FPS, was marketed by id Software for its "Realistic bullets, realistic blood, [and] real 3-D action,", in spite of the likely fact that most players of the game knew nothing of real blood and real bullets, only their depictions in cinema and television (as cited in Just Claws, n.d.). At the genre's inception, realism was already being levied importance, just as it continues to be through the refinement of the FPS as a genre.

One GoldenEye 007 (GE 007) television spot intersperses gameplay footage with footage from the film on which the game was based, and even makes a tactile comparison between the loading of the vibratory "Rumble Pak" into the system controller and the loading of a magazine into a handgun (Nintendo, 1995). The 
commercial is accompanied by the text "Where you direct the action...shot, by shot, by shot"; the player is thus positioned as both a combatant (James Bond) and the director of the film/game. This deserves further attention: the game is an adaptation of the film GoldenEye. Rather than situating the player solely as the avatar, in this case James Bond, the game also situates the player as the "director." Realism here explicitly relates to the aesthetics of cinema. GE 007 was also praised in the press for its realistic level design, weapon set, and movement mechanics (Perry, 1997). In an interview with Eurogamer.com, director Martin Hollis has pointed to realism as being of paramount importance in the development process and has even decried not being able to use the actual brand names of the weapons used in the game due to licensing issues because it would decrease realism (as cited in Whitehead, 2013).

Heather Loosemore, digital director of Starcom MediaVest Group, the marketing agency behind Halo: Combat Evolved, has chalked the game's financial success up to its immersive gameplay experience (as cited in SAY Media, 2011). The game is also featured in Daxgamer.com's “Top 10 Most Immersive Games Ever Made" list (Jorgensen, 2012). IGN.com's original 2001 review, giving the game a 9.7 out of 10, praised it for its immersive soundscape and stunning attention to graphical detail (Boulding, 2001). Xbox Nation Magazine in 2002 called the game "[...] quite simply the closest thing we have ever experienced to a living, breathing alternate universe" (as cited in Metacritic, n.d.). An interview with Pete Parsons of Bungie Studios is telling of the studio's goals towards immersive realism:

It's all about creating situational awareness or, from a marketing and PR standpoint, immersing you more into the game. But part of doing that is creating situational awareness. All of the sudden when you heard needles [...]it's like holy sh**! You sort of run and duck, and all 
of the sudden you start using the cover that we put there that you may not have used before. In the past, people just stood there and blazed away until they were dead or they ran away. Now it's more of a game of I'm going to crouch down, find cover and stand up shoot. It really changes the way you play the game. [...] So ultimately this unnatural fusion of the controller and the screen they all continue to melt away. (as cited in Valdes, 2004)

One television commercial for Battlefield 3 (BF3) intersperses gameplay footage alongside dramatized war footage and features the question "Is it real? Or is it Battlefield 3?" (EA DICE, 2011). Another blurs the distinction and offers a disclaimer that the gameplay footage shown is "Actual gameplay footage," as if it is so realistic as to not be obvious. It proudly displays a quote from Seth Schiesel of the New York Times calling it "The most realistic shooter yet" (as cited in EA DICE, 2011b). Stefan Strandberg, the audio director of the game, has put it plainly: "[...] I'm trying to make our games sound as immersive as I possibly can" (as cited in Tong, 2011). Unlike GE 007, the rhetoric surrounding BF3 (and to a lesser extent, Halo: Combat Evolved) refers to the reality of actual war in its aesthetic choices. The rhetoric surrounding the aesthetics of $G E 007$, likely because it is a game adapted from a feature film, refers to a sense of realism inspired by the cinema. Strandberg has described the influence of real warfare on the game's audio:

We're not going for a cinematic sound [...] It was a pretty easy artistic choice to go with a more documentary or more real version of the world. And I don't think you should aestheticize it too much. There's so much good in reality; there's so much iconic sounds that you as a player or you as a human being know is there. We don't want to manipulate that. People can decode the real world[...] (as cited in Game Informer, 2011)

The extent to which Strandberg's assertion that the game accurately reproduces the sonic reality of warfare is irrelevant however if we keep in mind the necessity of 
cross-media referentiality for the majority of game players. A gamer does not need to be a combat veteran to understand and appreciate the aesthetics of warfare as proffered by $B F 3$; they only need to be a veteran of war cinema, as has been noted.

To return to Chion (1994), it must be kept in mind that "realism" does not equate with "reality." Many complain that FPS video games are not realistic enough - Cheung (2012) offers one such example, when he complains of the use of heads-up displays and the inaccurate angling of the avatar's gun — but of course they are not. FPS video games do not strive to reproduce reality, to use Chion's (1994) language; they too, like cinema, are renders of reality, and present particular aesthetics of realism. To return to Wolf (1997), the precedent for reality in video games has been set by film and television; per Gieselmann (2007), for FPS video games, the precedent has been set by war cinema. While video games differ from films in some fundamental ways, as will be detailed at length, realism is thus designed in a similar manner. Realism, as a concept and goal, has been constructed discursively. In the case of the present sample, realism has been constructed in marketing by the game developers and in the critical reception. In general, FPS video games have been developed with the goal of immersive realism in mind - the blurred distinctions between reality and gameplay in the marketing surrounding $B F 3$ show this quite well. The other games at hand in this present study are no exception, as discussed above. But if immersive realism is the goal, how have experiences designed to promote immersive realism in the FPS been constructed? Before moving onto the question of immersion however, some further exploration of realism in the FPS is needed.

\section{$\underline{\text { Realism in play and interface }}$}


What does "realism" as concerns FPS video games comprise? What aspects internal to a video game contribute to its constructed realism? This section will attempt to answer these questions and, moreover, consider how designers have attempted to construct immersive user experiences specifically in the development of FPS games. How immersive realism is constructed sonically is the locus of this study, but realism in other, non-sonic, ways. This section will examine some of these. Chion (1994), discussing film, examined the sonic aspects of cinema. There is certainly no shortage of work concerning film, sound, and realism (Chion, 2009; Langkjaer, 2009; Metz, 1974). However, as Grimshaw (2007b) has noted, claims of realism concerning FPS video games have more often than not been constructed with graphical realism in mind - reviews, after all, feature a variety of graphical screenshots and clips of gameplay footage but very rarely, even in an era where embedding sound clips is no more difficult than embedding images, do they feature audio clips from games. But realism has been constructed in other ways, such as the narrative aspects of a game.

As Thomsen (2012) has noted, narrative realism can oftentimes be the most unsettling, as in various incarnations of the Call of Duty franchise which have featured historically accurate storylines alongside representations of political figures like John F. Kennedy and Fidel Castro. Anxiety-inducing, according to Thomsen, is the banality of interactivity; the player is thrown into a historical situation where its moral complexity has been stripped bare and replaced with the goal of winning the game. In this context, all actions, however gruesome, are heroic as long as they lead to successful completion of the game. And narrative realism does not need to necessarily reflect real historical figures or events, but is too found in details. As Thomsen has 
pointed to, seven Navy SEALs were punished for divulging military secrets concerning equipment and tactical procedures to EA Games during the development of Medal of Honor: Warfighter (Electronic Arts, 2012) in November 2012. Similarly, Baron (2010) has pointed to the historiographic effects of Call of Duty: World at War (Activision Blizzard, 2008), not just in its use of important World War II figures and its depiction of actual events, but in its portrayal of weaponry and attire from the period. Effectively, as Baron has noted, and similar to Thomsen (2012), the game and the player's interactions within — rewrites history, absolving moral complications and replacing them with the simple goal of defeating the enemy and winning the game.

Realism can also been constructed in the manners in which one interacts with the game. Virtual reality (VR) aside - console gaming, the object of study at hand, has little to offer towards a totalizing VR experience - human-computer interaction need not only take place via the abstraction of the "controller." Voice-over-IP (VOIP) communications are utilized heavily between squadmates in online FPS games, ideally, to strategize as a team, but more often than not they become the channel for racial slurs and homophobic epithets. The headsets donned by players, if not totally derived, are at least reminiscent in function to the various combat communications systems utilized by military forces around the world, such as the Modular Integrated Communications Helmet (MICH) favored by the US Army. But the recent Hyperkin ComRad follows also the form of these military systems. A hard plastic military style helmet with the choice of three camouflage patterns, the ComRad features built in speakers and an attached Bluetooth boom microphone and promises to "provide you 
an audio experience that makes you feel like you are truly in the game" (Hyperkin, n.d.).

Moving entirely from sound, other interfaces like gun controllers have been designed and marketed for their realism and promoted for their immersive capabilities. Avenger Controllers's Delta Six, modeled after a modern carbine assault rifle, promises an immersive experience for the player. Such controllers are certainly not new and date back all the way to the Magnavox Odyssey in the 1970s, but no gun controller, in a cursory review of the marketing and reception surrounding previous controllers, has promised an immersive experience in the same way that the Delta Six has. Avenger promises that "It doesn't get any more real than this" (Delta Six, n.d.). The controller, so real, they offer, has become the target of "delusional" anti-gun critics and thus must include an orange tip, the same sort found on other replica weapons like AirSoft guns. The controller, they add, may even run into international shipping issues due to its look and feel. Featuring realistic recoil and a variety of addon accessories (much like real military-grade assault rifles as well as the upgradeable weapons found in many FPS games, like $B F 3$ ), Avenger refuses to apologize for "delivering the most realistic, fun, and immersive gun controller on the market." And in the case that the prospective buyer does not understand just how realistic this gun controller is, amongst the one-sentence reviews from gadget and gamer blogs like Engadget.com and Gizmodo.com, they have even featured a "review" from CNN. Succinctly, it reads: "[The Delta Six] replicates the look and feel of a semi-automatic rifle." It is not so much a review of the controller's features and usability, as it is an affirmation, from a news source that features daily stories on warfare, that this 
controller is reminiscent of a real weapon. These materials and the ways in which they function as performative tools for the player call to light Nielsen's (2010) work on gaming as a somatic experience.

Outside of the material channels of interactivity such as controllers and microphones, interactivity — as the mode of engagement which separates games from cinema and other traditional media — perhaps lends the greatest possibilities for constructing immersive and realistic experiences. In addition to representations and narratives — narratologic realism — realism can also be constructed in simulative mechanics - ludic realism. Again, like aesthetic realism, simulative mechanics, in the case of FPS games, need not accurately portray real-world phenomena. They too are significantly preconditioned by prior media. For example, in the case of $B F 3$, its attempted implementation of real-world acoustical phenomena, such as dynamic reverb, does not need to be wholly congruent with the reverb of the real spaces being referred to by the game. Returning to Chion (1994), the reality of the phenomena merely needs to be suggested, and this is especially relevant given that most players of $B F 3$ have never actually shot a rocket-propelled grenade in either an enclosed space or atop a tree-less mountain; their only justifications for accepting the game's synthesized acoustic phenomena stem from prior media experiences. Sofge (2008), has interviewed Philippe Theiren, a designer at Ubisoft Montreal, to discuss the gun mechanics in Rainbow Six Vegas 2 (RSV2) (Ubisoft, 2008), a tactically minded FPS that for many is at the pinnacle of realism in FPS games. As Sofge has noted, Ubisoft went to great lengths to ensure the realistic representations of guns in $R S V 2$, from look to proper branding. Their mechanics, however, as Theiren has explained, are more the 
product of a constructed realism, one that adheres well to the aesthetic of realism in war cinema: "I take these weapons, and look at what defines them, or what people think defines them" (para. 5). In the examples he has given of the shotgun and the Uzi, their portrayal in the game is quite contrary to their real-world corollaries. While an actual shotgun has little penetrating power, in $R S V 2$ they can breach armor with little issue. Similarly, the Uzi, in reality a very reliable and accurate weapon, fires exceedingly fast and inaccurately. These depictions, as Theiren has described, are the product of assumed player associations — finding their origin in player interactions with previous media, from television, to film, to other games, and even fiction — and not supposed to be the accurate portrayal of real-world weapon mechanics. As Sofge has concluded, "If it feels like the real thing, game on" (para. 13). If it does not, he jokes, a recruiting station can always be found nearby. To return briefly to Chion (1994), a necessary step in engaging a work of cinema is accepting the rules of the "game," i.e., the conventions of realism as rendered rather than reproduced. Sofge's last point is well taken in the context of Chion: video games are not designed to replicate wholly the complex and external world at large. Technical constraints aside, it cannot be forgotten that video games, by and large, are entered with the assumption that they will be entertaining, fun, and functional. Sometimes the real world is none of these things. Games are meant to be played. Insofar as realistic representation and simulation interferes with play, the experience moves from that of a video game to that of a pure simulation. While both may offer immersive experiences, this study in concerned with games. But what exactly is immersion and what is the relation between immersion and realism? 


\section{$\underline{\text { Immersion }}$}

As Grimshaw, Charlton, and Jagger (2011) have noted, immersion and realism are allied. As has been argued, FPS video games have been designed in the pursuit of immersive realism, and the innovation and implementation of new audio reproduction technologies manifests this. But what exactly is "immersive realism"? Realism, as it has been discursively constructed in the texts surrounding particular games, has been described. But what of the other half, immersion? This section will interrogate the degree to which they can be considered allied concepts, explicating immersion in a general manner, apart from the sonic aspects of FPS video games. The latter will be taken up at length in the following sections.

\section{$\underline{\text { Immersion and video games }}$}

Immersion has been defined in a multitude of ways, and concerns multiple objects of study. This section will proceed through some of this literature. Apart from how video games can construct a sense of immersion, immersion has been theorized at length as it concerns presence and telepresence in VR and cyberspace. Reiner and Hecht (2009) have proposed a particularly succinct, and conventional, definition of VR presence which understands immersion as occurring as the interactant loses a sense of physical, bodily presence and instead finds themselves extended into the virtual environment. As Grimshaw, Charlton, and Jagger (2011) have noted, such a definition can be traced back to Denis Diderot, who in the eighteenth century theorized a similar sense of presence in the viewing of a painting: in attending to a work, the beholder can lose a sense of self, removed to the world of the painting. 
Extending this definition from VR to video games, Ermi and Mäyrä (2005) have formulated one of the more widely accepted conceptualizations of immersion. Similar to Reiner and Hecht's (2009) definition of presence, Ermi and Mäyrä also understand immersion as occurring in the dissolution of the self outside the game and a recognition of the self as either physically or virtually part of the mediated experience. Their study has analyzed how immersion occurred in thirteen games and in conclusion they have posited three types of immersion: (1) sensory, where immersion occurs as sensory stimuli in the game override sensory stimuli in the physical environment; (2) challenge-based, where immersion occurs in the use of motor and mental skills; and (3) imaginative, where immersion occurs in identification with game characters. Continuing with video games, McMahan (2003) and Grimshaw (2007a) both have considered how immersion and realism function in tandem. For McMahan (2003), and similar to Ermi and Mäyrä (2005), a sense of immersion is constructed both in realistic representation (in the sense of authentic and not realism as discussed in the preceding section) and the player's interactions with the environment of the game; the two must be carefully balanced to produce the greatest sense of immersion. Grimshaw (2007a) understands the balance between representation and interaction in a similar manner, but instead of authentic reproduction in graphics and audio, argues that a "reduced" realism, "operating at the level of convention, consistency and verisimilitude," has the greatest potential to immerse the player (p. 123).

In their examination of the varied literature on presence and immersion in VR and video games, Grimshaw, Charlton, and Jagger (2011) have found three common 
threads: (1) the player must have a sense of being in the world of the game; (2) concerning interactivity, the player must have a sense of acting in the gameworld; and (3) both the sense of being and the sense of acting are tied to the perception of realism, which is less reliant on strict authenticity and instead constructed as a sense of verisimilitude. It is in this manner that immersion will be discussed throughout the remainder of the present study.

As Reyes and Adams (2010) have note, inculcating a sense of immersion in the player — via the design of the user experience — is only justifiable (and necessary) when a boundary that would otherwise inhibit immersion must be overcome. Immersive experiences are not spoken about concerning games taking place in the "real" world; players in the NFL are not given acclaim for their presence, nor do they comment on the degree to which they feel immersed in the game, largely because they are physically in the game. The player of a video game, however, to return to Reyes and Adams, is screened from the game. Whether literal, such as the LED television utilized in this study, or figurative (even a game of pinball is screened via the glass covering and spring-loaded rod), the screen serves to isolate the player from the field of play. This screening, as a function of the game apparatus, limits user interaction implicitly; again, unlike a game of football where, while rules exist, they can be very easily broken and in the most absurdist of ways, in screened play the screen separates the player from the field of play and thus affords certain manners of interaction at the expense of others. Screened games, such as video games, are defined by limitation. As Reyes and Adams have made clear, it is both the limiting of input by the user and the limiting of feedback from the game that construct "the primary conditions of 
possibility for screen-play as such" (p. 150). To return then to immersion: via the screen, via the limited input and the limited feedback of the game apparatus, the player is estranged from the field of play. In the virtual world of the video game, the goal of constructing an immersive experience is thus to overcome, at least temporarily, this estrangement, and to extend the player's very real body into virtual space, while still maintaining those aspects which keep the virtual world a game. It is in this extension that the player is reconstituted as subject in the world of the game. Immersion can occur in a variety of ways, as has been briefly demonstrated to in this section. Retreading the questions that began this section, concerning "immersive realism" and the degree of alliance between these terms, it is this present study's contention that one particular way designers have attempted to craft immersive experiences in FPS games is through utilizing the aesthetics of realism as found in war cinema and the incorporation of functional, simulative mechanics, all designed to briefly overcome the estrangement of the screen. Realism has been discussed as it has been constructed external to the game-text, in the discourse surrounding games, from marketing to critical reception, but how has immersive realism been constructed through FPS audio?

\section{Immersion and FPS audio}

While immersion and realism have been discussed in a general manner surrounding video games, this section will explain more deeply how sound is used to construct immersive experiences in FPS games. What has the most obvious potential for immersion in the FPS is its central aesthetic quality: its first-person perspective. The first-person visual perspective of these games positions the player, not as a puppet 
master controlling an avatar as in a third-person perspective, but as the avatar.

Visually, the player is present with their virtual arms and hands that are holding their weapon. The player is positioned within the body they are controlling and navigating the present virtual world with. This designed identification with the eyes of the avatar positions a similar identification with their ears. The following discussion approaches the user experience of FPS games at this point.

Grimshaw, Charlton, and Jagger (2011) have argued fundamentally that insofar as FPS game audio relates to lived experience, it is most capable of providing possibilities for immersion. As Chion (1994) has argued concerning film sound, it is not necessary that reality be reproduced, but merely hinted at. As Grimshaw, Charlton, and Jagger have made clear, in the use of 3D audio in sound design, made possible in the early 1990s, game audio becomes more capable of approximating lived experience. Real-world sound emanates from a 360-degree sphere and this can, at the very least, be approximated by contemporary game audio engines. Game graphics on the other hand, no matter how advanced, are still presented on an empirically $2 \mathrm{D}$ screen. VR still very much a niche market, contemporary console games still output to screens affording no perspectival differences than those of their 1960s and 70s predecessors. Coupled with the possibilities for realistic acoustic effects such as reverb, echo, and various types of modulation and the possibilities to interact with sound-producing objects and in sound-producing events in real time, contemporary FPS game audio provides a variety of ways in which the possibilities for immersive experiences can be constructed. Of course, as Grimshaw, Charlton, and Jagger have concluded, and to return to my previous discussion, immersion is not necessarily 
predicated on exacting simulation and the reproduction of reality. Game audio, however advanced it may be in 2013, is still not capable of duplicating the richness of real-world soundscapes, and yet, can still provide for immersive experiences. As has been discussed at length in this study, it is not necessary the goal of FPS game developers to accurately reproduce acoustic reality — again, most gamers are not combat veterans and would not even notice if a game did accurately reproduce the sound of C4 explosives blowing apart an M1 Abrams tank. Rather, game audio in the FPS is designed and implemented in adherence with the aesthetics of realism as constructed in previous media like war cinema; it is a remediation of such cinematic reality. While most players have not experienced the close-range firing of a fully automatic weapon, they have heard these sounds rendered, to use the language of Chion (1994), in films. Thus, their familiarity and ensuing literacy is with the constructed reality of cinematic audio, which has come to stand in for actuality. This point is taken up at length by Grimshaw (2007a) in an earlier work.

As Grimshaw has noted, game audio exists, at least that which seeks to be representational, on a continuum between mimetic realism and abstract realism. The former concerns simulative realism while the latter concerns emulative realism. As has been discussed at length throughout the present study, an immersive experience is not predicated on the former. According to Grimshaw, a "reduced" sense of realism, rather, is all that is necessary. He has provided a particularly relevant quote from James Lastra: "Decades of tin-sheet thunder and coconut shell hooves prove [...] that fidelity to source is not a property of film sound, but an effect of synchronization" (as cited in Grimshaw, 2007a, p.147). In video games too, immersive 
realism is less a product of mimesis and simulation and more a product of synchronization and verisimilitude, adherent to the concept of "realism" as it has been discursively constructed in the aesthetics of film and other representational media.

Concerning more directly how game audio can be utilized to construct the possibilities for immersive experiences in the FPS, Grimshaw has applied Ermi and Mäyrä's (2005) three types of immersion: sensory, challenge-based, and imaginative. The first, sensory immersion, refers to the ways in which game stimuli may override stimuli from the surrounding environment where gameplay takes place. Sensory immersion is physical immersion that occurs beyond the virtual world. For Grimshaw, tying sensory immersion in with game audio is the most obvious; game audio is sensually immersive when it obfuscates sounds external to the game and is thus largely dependent on the type of audio interface utilized by the player and the formal qualities of the sounds reproduction. Simply enough, tinny, quiet two-channel computer speakers are less likely to be sensually immersive than an 8.1 surround sound system or studio-quality headphones. Challenge-based immersion concerns immersion as it occurs via the use of mental and motor skills. In the FPS, Grimshaw has understood weapon sounds, as they might indicate an impending threat, and status signals such as those that indicate when a player receives ammo or when a teammate is killed, as capable of producing challenge-based immersion. Finally, imaginative immersion, as it occurs in player identification with story and characters, might take place sonically in the FPS via dynamic bodily sounds, such as breathing rates that vary with activity intensity and realistic footsteps. 
Huiberts (2010) has similarly examined Ermi and Mäyrä’s (2005) types of immersion in his account of game audio, albeit apart from FPS games. In a general manner, game audio may provide the possibilities for sensory immersion in any manner of ways in that its audio constitutes a world and provides the player with a sense of presence in that world. At the same time both sensory and imaginative, a game may be immersive as it constructs an overarching atmosphere that draws the player within, via ambient sound and music. Furthermore, a game may provide an imaginatively immersive experience as it creates emotional change in the player, likely via ambient sound or music. Finally, a game may provide a challenge-based immersive experience via audio that inspires a focus on stealth tactics or audio that is anticipatory or stimulates tension.

Ermi and Mäyrä's (2005) three types of immersion will be returned to in the following sections, where the present sample of games will be explored per how they position the player-subject in the sonic regime of the game. Presently however, now that what constitutes immersive realism has been explored at length, the history of video game platforms will be examined, from the earliest days of video games in the mid-twentieth century, to the release of the PS3 in 2006, with the goal of evidencing how technological innovations have come about coupled on a trajectory with the pursuit of immersive realism as a design goal.

\section{Platform history}

The history of video games — as a technological history, to return to Juul (n.d.) - is also a discursive account of how the aesthetics of immersive realism have come to be constructed. It is this paper's contention that the impetus behind 
technological innovation, and specifically innovation concerning audio reproduction, in video game platforms has been in a large part the drive towards more immersive, more realistic, gameplay experiences. However, this innovation takes place in the context of a major marketplace, and it must also be kept in mind that the goal behind increasing the capabilities of systems is not necessarily driven by the pure goal of increasing immersive gameplay experiences, in the case of FPS, for their own sake. Like any mass media, but especially so for console gaming, innovation is also driven by market differentiation, by setting up one product as superior to others. While, for example, increasing the capabilities for real-time digital signal processing (DSP) may help to promote an immersive gameplay experience, or at least what has come to be called an immersive gameplay experience, it also sets a console apart from its contemporaries that might not offer such capabilities. Where the goals of market differentiation and of constructing immersive gameplay experiences intersect is where this study approaches games. Before moving on to a turn-by-turn breakdown of the audio reproduction technologies for each platform espoused in the sample, I will begin with a brief historical account of the inception of video games as particular, purposebuilt technological systems aimed in many cases at constructing realistic experiences. The goal is to elucidate their capabilities as they pertain to the construction of sonically immersive interactive experiences.

\section{Pre-history}

In attempting to construct a historical account of video games prior to the systems at hand, the aim is not in comprehensiveness — that has been done at length (Dillon, 2011; Donovan, 2010; Kent, 2001; Melissinos \& O'Rourke, 2012; Wolf, 
2012) - but in explicating various technological advances prior to the release of Nintendo's SNES and the assumptions, goals, and practices from which they originated. At the core of this discussion are two questions: (1) in the history of video games prior to the SNES how had realism been thought about, and (2) how had designers sought to construct realistic gameplay experiences? The section borrows much of its historical information from Donovan's (2010) work surrounding video games. Throughout this section, it should be kept in mind that, as Juul (n.d.) has written, the dual histories of video games and the technology of their representation should not be thought of causal in a unidirectional manner, but rather understood as mutually influential; innovation begets new games and new games beget further innovation.

Conceived in the context of the early Cold War, video games find their origins in the late 1940s and 50s, tied in inextricably with the "Space Race" between the United States and Soviet Union and the greater history of computing and artificial intelligence. Throughout this time, a variety of simple games were conceived and attempted (not the least of which by groundbreaking computer scientists and mathematicians, Alan Turing and Claude Shannon), but computing limitations, both technologically and in terms of machine timesharing capacity, constrained the possibility of the complex mechanics necessary to produce a digital game. In 1962 however, a group of MIT students coded Spacewar! on a DEC PDP-1. The PDP-1 was eminently capable with its improved cathode-ray tube (CRT) display and keyboard input, as well as greater and more efficient memory, compared with its predecessor from Texas Instruments, the TX-00. Gameplay mechanics of Spacewar! were simple 
but novel for the time and even included an attempt at realistically simulating the gravity field of a star. Devised by a group of self-espoused science fiction fans, Spacewar!, designed for two-player play only, involves two armed spaceships which must attempt to shoot one another while navigating this gravity field. Graphics are simple CRT line drawings and audio is non-existent. As Donovan (2010) has noted, Spacewar! came at a time when televisions could be found in 90 percent of households, up from the minuscule .5 percent immediately post war. Spacewar! of course could not be played on televisions and instead required prohibitively expensive $^{2}$ and esoteric machines. However, as the game was spread from university to university and the ubiquity of televisions at home increased, the seed was planted. The preconditions for the viability of the video game as a mass-market medium were in place.

Concerning realism, it is worth noting that Spacewar! was designed not only with the goal of incorporating realistic space physics, but moreover was an attempt at simulating something both very relevant and very real in the 1960s: space travel. The same year that work on the game was begun at MIT, both Soviet cosmonaut Yuri Gagarin and American astronaut Alan Shepard made the first two spaceflights. Popular culture was abuzz with all things "space," not the least of which was science fiction. In that the game attempted to reproduce the physics of space travel, it sought to reproduce an already existent reality, or at least an easily conceivable reality found in the aesthetics of science fiction. Thus from their very inception, video games have been intertwined with simulations of reality or conceivable realities. This is not to say that video games not dealing with realistic simulation (like a version of tic-tac-toe

2 : The PDP-1 cost $\$ 120,000$ in 1960 , as Thelen (2004) notes. 
programmed on the TX-0 computer at MIT in 1959) are not important in the development of games as a medium. They were and still are. Yet, realistic simulation from the start was a goal; it never had to be but it was, and this is of prime importance in understanding the historical discourse surrounding realism and video games as well as the impetus for technological innovation in FPS game design.

While technological limitations continued to constrain the possibilities for realistic simulation in games themselves, supplemental materials, from peripherals, to, box art, to arcade cabinet design, in the early history of games, aided in defining what the game was about and, moreover, in the era of simple graphics, what the various moving geometric shapes and 8-bit colors were representing. The Magnavox Odyssey, the first digital video game console, was released in 1975 and was sold complete with various transparent plastic overlays that could be applied atop the user's television screen in order to augment the system's primitive graphics. These overlays provided the backdrop for many games, from a basic racing simulator to tennis. While empirically in the foreground, these overlays are meant to be experienced as background. The "real" of the primitive graphics is replaced with the aesthetic of realism, literally applied to the screen. Similarly, the cabinet art for various Atari, Inc. arcade titles as well as box art for Atari 2600, released in 1977, cartridges promote realistic representation and imply the need for a major suspension of disbelief on the part of the player — the basic gameplay mechanics of Pong (Atari, 1972) after all have been used to represent games beyond just tennis, including handball, soccer, hockey, and even basketball and volleyball. In an era where the most advanced graphics available consist of colored 8-bit lines and blocks, a certain level of 
imagining is necessary on the part of the player if they are to play the game as depicted on the box and implied in the title. The box art of these games, specifically of Atari 2600 games, provides the visual vocabulary of the game, in vivid hand-drawn detail. Webster (2013) references a quote from Tim Lapetino, currently working on a book about Atari 2600 art: "Part of what made the world complete was the artwork that conjured up this other place. I wasn't sitting in my living room anymore; I was on this desolate planet or in space. And it was mostly because of that art" (para. 2). In lieu of a visually immersive gameplay experience, box art served to draw the player into the virtual world, including a world as abstract as Breakout (Atari, 1976) or even a tutorial for learning the BASIC programming language.

In addition to Atari's attempts at crafting immersive gameplay experiences through supplemental art, the Atari 2600 also heralded one of biggest technological breakthroughs in video game design since the release of Spacewar!: the microprocessor. Prior to the incorporation of microprocessors in game design, games were designed largely as hardware. The development of a game necessitated careful hardware design. The microprocessor introduced the possibility to program — and, most notably, re-program — games as software. Consoles became cheaper and smaller without all of the necessary components and meticulous wiring necessary to do the work of the microprocessor. The possibility was opened up, with the new affordances of re-programmable systems and greater computing power, to focus on bettering the graphical and, to a much less extent, sonic aspects of games. Video games are complex. Even a game as simple as Spacewar! required 200 hours of programming time on the PDP-1 (Game Developers Conference, 2013). In terms of raw computer 
power, however, the latest Intel processor for home desktop use, the Core i7 Haswell has 1.4 billion transistors (Shimpi, 2013) compared to the PDP-1's, expansive at the time, 2,700. Video games, and the technology deeply intertwined with their production and reception, have evolved in the pursuit of complexity. This complexity may take the form of immersive realism, as it does in FPS games and other simulative genres, or it may take on more abstract form such as in puzzle and strategy games (which arguably seek to provide immersive experiences in ways different than audiovisually realistic games like FPS games). The introduction of the microprocessor in the mid 1970s opened the floodgates for further developments in this vein. A quick overview of just a few such developments should suffice in elucidating this drive to innovation.

Four years after the microprocessor, two further innovations in game development would help to usher in a new era of creativity. According to Donovan (2010), both high-resolution vector graphics and color graphics offered newfound possibilities to craft immersive gaming experiences. Ed Logg, a programmer at Atari, has discussed these possibilities in regards to Atari's Asteroids (Atari, 1979):

Vector monitors are high resolution. They are 1064 by 728 pixels whereas standard rasters are 320 by 240 - a big difference in resolution so when you turn your ship you can tell which direction it's facing, which is really important. (as cited in Donovan, 2010)

While using graphics to navigate in a virtual world may seem obvious, if not banal, in the contemporary era of gaming, at the time this innovation was profound. Equally profound was another possibility afforded by vector graphics: 3D visuals. Far removed from what counts for 3D in cinema, 3D game graphics imply approximated three dimensionality in 2D space, like normal cinematic visuals, though accomplished via real-time rendering and more complex animation. In most cases, this involves the 
possibility for real-time player-controlled perspective. Atari released Battlezone (Atari, 1980) in 1980, complete with 3D vector graphics. The game's first-person perspective - from the driver seat of a tank — was also novel for the time, so much so that, as Donovan (2010) has noted, the US Army requested Atari design a simulator for their Bradley Infantry Fighting Vehicle (a protoype was completed but the project was scrapped). With its simplistic wireframe graphics, the 3D visuals of Battlezone were ahead of their time. The heyday of 3D wouldn't come for a decade, but these innovations combined were enough for Trip Hawkins, the director of strategy and marketing at Apple in 1982 who eventually go on to found Electronic Arts (EA Games), to foretell a coming time when video games would be a major consumer market. Thinking back to his first encounter with video games in 1972, Hawkins saw the immersive potential of games: "I had a strong feeling that people were meant to interact, not to sit passively like plants in front of the TV. I was already designing board games but saw instantly that a computer would allow me to put 'real life in a box"' (as cited in Donovan, 2010, p. 138). Innovation continued throughout the 80s: processor speeds increased, augmenting their available computational load; memory capacities went up; digitization of real-life action and sound became possible; and, in major shift, via the work of Jay Miner and Amiga, the first graphics processor was developed, independent of the microprocessor. The importance of this last shift cannot be understated: a standalone graphics processor freed up much of the resource strain on the microprocessor and allowed for greater complexity both in graphics, but also in the other calculations necessary in the coding of a game. Commenting on the decade as it came to a close, Eugene Jarvis, designer of arcade classics such as Defender 
(Williams Electronics, 1980), saw a coming boom, like Hawkins: "I could see that technology was evolving beyond the pixel-based artwork of the '70s and early ' 80 s towards 3D animation, motion capture, digitization, you name it. The video game field was about to explode in a technological big bang enabling immersive and rich gaming as never before seen" (as cited in Donovan, 2010, p. 151). And the 1990s delivered.

While the 1990s brought a variety of technological innovations in game development, most notable for the study at hand is the continued refinement of 3D visuals. As the 80 s ended and the 90 s began, VR took hold as a design goal for many game companies. With countless research projects and a variety of start-up companies, many sought to bring VR, which conceptually had existed since the 1960s, to the mass-market game world, beyond the research lab and into the home. Yet, there was no existent consumer market for VR; Virtuality changed that in 1991 with Dactyl Nightmare (Virtuality, 1991), an arcade VR experience. Each rig cost $\$ 65,000$ and consisted of a head-mounted display, a joystick, and a wearable glove controller. While the game was featured in many arcades, the cost disallowed extensive play sessions and limited users (in four-player sessions) to only a 3- or 4-minute experience in order to satisfy the lengthy queue. Yet the game left its mark, both on the industry and its players. Players wanted more VR experiences, but game companies realized the inability to provide these in the home given the prohibitive costs. Instead, resources were delegated to designing immersive console-based experiences through the refinement of 3D graphics. While 1980's Battlezone featured 3D visuals from the first-person perspective, its wireframe style was primitive when compared with the graphical possibilities of the early 1990s. Moreover, unlike 2D graphics which were 
pre-drawn, 3D graphics are drawn in real time and thus more tolling on system resources. However, with the technology available in the early 90s, this problem became readily manageable. id Software, in 1991, tackled the challenge.

In the context of this industry attention to VR, Chris Green, a programmer at Looking Glass Studios, devised a novel way of drawing 3D graphics. His engine was far more efficient than previous attempts and in addition was able to wrap 3Dgenerated polygons in textured patterns. Thus, while early attempts at 3D necessitated a major suspension of disbelief on the part of the player who must take a wireframe structure or a solidly colored block as a brick wall, Green's engine allowed a 2D drawing of brick to be applied to the polygon structures comprising a wall. Meanwhile, id Software, a developer with greater resources than Looking Glass, in 1990 was beginning to pursue 3D graphics and programmer John Carmack was charged with replicating Green's engine. In 1991 they released Catacomb 3-D (id Software, 1991), a first-person dungeon game. The game was a hit and id decided to focus most of its resources in the 3D realm. One year later they released Wolf $3 D$ for the personal computer, credited as the very first FPS and of which a port is part of the sample of the present study. The game was revolutionary, as Donovan (2010) has noted: "[It] raised the standards for 3D visuals to a new level, forcing other game designers to rethink their work" (p. 258). Continuing to push the envelop, Carmack rewrote his 3D engine and in 1993 the studio released Doom, a science fiction FPS, complete with the capability for online play as well as map editing. If Wolf $3 D$ spawned the FPS, Doom popularized it. It was a paradigm shift, as Donovan has noted, and "video games were never quite the same" again (p. 261). The move from 
2D to 3D graphics did not create a new plane of representation; graphics were still reproduced, empirically, in two-dimensions, just as in cinema. However, in creating the illusion of real-time player-controlled perspective, especially as this could be coupled with a first-person perspective, 3D graphics greatly augment a game developer's ability to construct an immersive gameplay experience vis-à-vis the rendering of vision, a rendering that in its interactivity, goes beyond that of cinema's. But moreover, as will be discussed, 3D graphics open up the possibilities for 3D audio. While true VR was not possible due to technological limitations at a massmarket scale, this approximated 3D was both possible to represent efficiently and produce at no additional cost on the present hardware. Thus, to apply the lessons of Sterne (2003) to the present material, not only did what counts for immersive and realistic in cinema help to construct what would count for an immersive gameplay experience, but so to do did the present technological limitations as well as what the market could bear as a commodity.

From Spacewar! to Doom, the history of video games from the 1960 s to the mid 1990s has been in large parts a history of technological innovation. In the pursuit of increased complexity in the mechanics of games, developing increasingly complex means of game development and representation, such as 3D visuals, became necessary. As concerns the present study, in many cases "complexity" was intertwined deeply with the representation and simulation of realism. Between the simple visuals of Spacewar!, the box art of Atari 2600 games, the growth of 3D graphics and VR, and beyond, realism has been a core focus of game design, and the goal of totally immersing players in the virtual world of the game has been the impetus behind much 
technological advancement. The discourse surrounding realism, as demonstrated earlier in a review of the marketing and critical reception surrounding these games and consoles, weaves threads throughout the history of video games, and takes particular material existence in the technologies developed in their development. While this section has attempted to summarize the technological history of games, the proceeding will more closely examine the technological history and intertwined capabilities of four particular systems: the SNES, the N64, the Microsoft Xbox, and the Sony PS3. First, however, a more specific examination of four particular technological developments pertinent to the construction of immersive experiences via game sound will be examined.

\section{Immersive realism and technologies of audio reproduction}

While the preceding section spoke largely of technology as it concerned graphical representation and mechanics, the present study is concerned with technologies of audio reproduction. As has been noted, the history of video games has in large parts expressed a particular visual bias. In their development, marketing, and both player and critical reception, audio is often treated as secondary to graphics. However Collins (2008) in her history of video game sound, music, and the technologies of its reproduction has noted that audio is no less important in developing an immersive (and entertaining) video game experience. Throughout her book she has exemplified four particular technological developments that had particular influence on this end: (1) newfound possibilities for three-dimensional (3D) sound; (2) newfound possibilities for real-time DSP; (3) newfound possibilities for an increasing number of possible simultaneous samples; and (4) increasing bit depth and sample 
rates of digital audio samples as well as the increasing availability of system resources.

3D sound came about in the 1990s, influenced greatly by cinema sound. Whether simulated in a two-channel speaker setup or reproduced via an analog or digital surround sound setup, 3D sound increases possibilities for player immersion by reproducing the 360 degree field of sound that a player would hear in real-life experience. The evolution from mono to stereo sound was important in that it allowed sound to be distributed about a player's visual field at any given time (180 degrees). The evolution from stereo to surround sound however allowed the possibility of feedback from objects and events located behind a player, and in a way quite natural, unlike graphical analogs which might allow perspective switching or utilize textual feedback. Moreover, fore and aft feedback could be ascertained simultaneously, again a feat quite awkward and unnatural via visual representation. While perception, as screened via the gaming apparatus, had traditionally been unidirectional, the development of 3D audio capabilities allowed stimulus to be localized in a multitude of simultaneous fore-aft and port-starboard positions. This is especially important given the limitations of 3D graphics, as have already been noted. Given that 3D graphics are still empirically 2D, the possibilities for 3D sound greatly augment the possibilities for presence in a world; the virtual world literally comes to surround the player. Coupled with real-time DSP, the effect can emulate real-world audition quite accurately.

Real-time DSP came about in the early to mid 2000s. The evolution of preprogrammed DSP in video games, as Collins has noted, is deserving of a book itself, 
but the possibilities to encode DSP in real time were revolutionary. DSP includes adding effects like reverb, chorus, echo, filtering/equalization, amplitude adjustment, panning, time stretching, and attack-decay-sustain-release (ADSR) envelopes (used in instrument and human voice synthesis). Apart from the real-time synthesis of these effects, adding, for example, a generic echo to a player's footsteps in the setting of a virtual cave increases a sense of realism by emulating approximately how the acoustics of a cave might affect real-world sounds, or least how a cave often affects sounds in cinematic representation, which are not necessarily congruent. Without processing in real time, such an echo wouldn't be variable; while the game might recognize a player's presence in a virtual cave and insert a pre-programmed sound accordingly, it cannot alter this sound as the player moves about the cave in a truly variable manner. Moreover, without the concurrence of 3D audio, these echos will only be located in front of a player. The development of real-time DSP allowed this echo to be modulated as the player navigated the virtual world, altering perhaps the delay time or feedback dependent on the shape of the player's immediate space and any obstructions to the sound signal, from stalactites to non-player characters. The possibility to emulate real-world acoustics lends itself to quite well to immersing the player in a virtual soundscape. Yet, even when conflated with the possibilities for 3D sound, these technologies offer little for the construction of an immersive gameplay experience if they are only applied to a single sound at a given time. As the possible number of simultaneously reproduced samples increased, so did the possibility of crafting a rich and variable sonic world. 
Early video game sound could only reproduce a couple samples of sound simultaneously, dependent on the amount of channels for sound offered by the platform's sound processor. The Atari 2600, as Collins (2008) has noted, could only reproduce two, which were then mixed down into a mono output. In a real-world soundscape, innumerable sounds occur simultaneously — in the office where this study is presently being written, the gentle hum of computer fans, two officemates typing, a beeping elevator from far way, a distant truck in reverse, muffled voices from floors above and below — the list could go on. In most day-to-day experiences, there is always present an immense simultaneity of occurring sounds, sounds that interact with the acoustics of the immediate space and sounds that are heard in a complete 360-degree sphere. By augmenting the breadth and depth of a virtual soundscape, a sonic world more akin to the player's non-virtual experiences of hearing can be created, minimizing the limitations created by the screen. However, as Sterne (2003) has made clear, recorded and synthesized sound are not duplicates of their "original." The apparatus, whether it be the game engine where real-time DSP occur or the manner in which sound effects were recorded in the field or studio, always leaves traces on the copy. As concerns digital audio, which all game audio is, these traces can be the analog artifacts left by the recording apparatus, but more relevant to the reproduction of digital audio, they include the bit depth and sample rate of the present sample(s). Hopefully a brief example will suffice.

Even if a game engine offered the possibility for tens of thousands of simultaneous samples, if the bit depth was only 4-bit and the sample rate was 8,000 $\mathrm{Hz}$, the experience would be far from realistic, as the reproduced sound would contain 
an unbearable amount of digital artifacts. Without detailing the complex specifics of why this is so, essentially the result would compare to a poor-quality digital image. To preserve an analog sound digitally, it must be converted; a physical quantity, in the case of sound, amplitude, must be quantified digitally. This is the ADC/DAC process — analog-to-digital conversion and its inverse, digital-to-analog conversion. Ignoring many subtleties, this process begins with "sampling," which involves the converter taking regular measurements of a sound wave's position at a given time — the "sample rate". These amplitude measurements are quantified digitally through "bit depth." While not entirely analogous, sample rate and bit depth could compare to pixel resolution and color depth, or more simply, how pixelated a digital image is and how many (or how few) colors it can represent. A pixelated image in greyscale is not very helpful in promoting an immersive gameplay experience; in terms of audio, nor would sound reproduced at a sample rate of $8,000 \mathrm{~Hz}$ with a 4-bit depth. The higher the bit depth and the faster the sample rate, the more "accurate" and higher quality the sample. Early systems like the Atari 2600 were 4-bit, while current-generation gaming systems like the PS3 and Xbox 360 capable of reproducing audio at 24-bit bit depths over 24-bit tend to produce little to no audible difference (Applied Acoustics Systems, n.d.). Concerning sample rates, many early arcade systems as well as early consoles like the Nintendo Entertainment System and Sega Genesis, while capable of higher, were optimized to reproduce audio at 22,050 kHz; currentgeneration consoles like the Sony PS3 and Xbox 360 are capable of up to $192 \mathrm{kHz}$, but in most cases audio is reproduced at $44.1 \mathrm{kHz}$ (the CD-quality standard) or $48 \mathrm{kHz}$ again, like bit depth, there is a certain threshold where sample rate differences are no 
longer audible and may even introduce undesirable artifacts into the audio stream ${ }^{3}$ (Applied Acoustics Systems, n.d.).

Combined with increasing processor speeds, the development of graphics and audio processors separate from the main console CPU, increasing memory capacities, increasing storage space on the media itself (i.e., in the shift from cartridges, to CDROMs, to DVDs, to Blu Rays), and on the console, and in the development of cloudbased storage, the above four developments increased the possibility for designers to crafts immersive and realistic gameplay experiences in novel ways. Of course, the influence was not unidirectional. In that the impetus in game design, at least as concerning audiovisually realistic games, was towards greater immersive realism, then this impetus was also partially behind the growth of these very technologies. With the above history in mind as well as Collins's (2008) four pinnacle developments in game audio, a turn-by-turn account of the audio capabilities of the four consoles utilized in the present sample will provide the necessary foundation to examine how these technologies have been utilized in constructing immerse and realistic experiences in FPS games and moreover how these new experiences have (re)positioned playersubjects diachronically.

\section{Super Nintendo Entertainment System}

Nintendo's SNES was released in 1990. Aside from its innovations in 3D graphics, the SNES featured a self-contained audio subsystem, allowing more system resources to be devoted to audio reproduction. Among other components, the audio subsystem consists of an 8-bit co-processor (the SPC700) and a 16-bit DSP processor,

\footnotetext{
${ }^{3}$ There is some oversimplification here. While bit depths over 24-bit and sample rates over $48 \mathrm{kHz}$ may offer no audible difference, they do provide more information for the game engine to act upon, potentially.
} 
both manufactured by Sony who would go on to produce the Playstation console. The 16-bit DSP is capable of mixing 8 independent samples with a 16-bit bit depth and at $32 \mathrm{kHz}$, all output in stereo sound. Effects, not available to be processed in real time, include echo, delay, reverb, ADSR envelope control, and panning.

\section{Nintendo 64}

The N64, released in 1996, and featured the most powerful processor among consoles of its generation, a 64-bit NEC VR4300. This main processor, along with RSP (reality signal processor) controlled both game audio and graphics. Audio was output in 16-bit stereo (though some games did support surround sound) and as Collins (2008) notes this could be potentially enhanced by Evergeen's third-party RumbleFX 3D Sound Amplifier add on, which promised to simulate surround sound on a stereo system. The system was capable of sample rates as high as $48 \mathrm{kHz}(44.1$ $\mathrm{kHz}$ is CD quality) and in a giant leap from Nintendo's previous system, the SNES, supports 100 possible simultaneous samples (though considering the limitations of the CPU and storage, most games only used about 16 - 24 on average). Compared with Sony's competing system, the Playstation — released in 1994 - the 64 was more capable in terms of possible channels and sample rates. Moreover, the system supports real-time DSP effects such as reverb, chorus, and panning. However, as with sample rates and simultaneity of channels, in practice reproduction was limited by system resources.

\section{$\underline{\text { Microsoft Xbox }}$}

Microsoft released the Xbox in 2001, largely in competition with Sony's Playstation 2, though Sega's Dreamcast and Nintendo's Gamecube both occupied a 
significant market share. In addition to innovations in graphics and storage that outclassed competing systems, the Xbox's audio subsystem was significantly more capable than its contemporaries. Processed external to the main CPU, NVIDIA's SoundStorm offered the possibility of 256 stereo samples or 64 3D surround sound samples, with sample rates as high as $48 \mathrm{kHz}$ and bit depths of 24 bit. As with any system however, audio reproduction is limited by by available system resources 256 24-bit simultaneous samples at $48 \mathrm{kHz}$ would be impossible — but careful resource allocation could allow for well-developed, dense soundscapes, complete with a variety of real-time DSP filters.

\section{$\underline{\text { Sony Playstation } 3}$}

Sony's PS3 was released in 2006, in competition with Microsoft's Xbox successor, the Xbox 360, and Nintendo's Wii console. The PS3's main CPU, dubbed the "Cell," is responsible for most console functions as well as audio and graphical reproduction, thus necessitating careful balance of resource allocation. Concerning audio, the system's capabilities demonstrate immense growth from Sony's previous Playstation 2, including up to 512 possible stereo channels of sound as well as sample rates as high as $192 \mathrm{kHz}$ via HDMI audio. Fully surround sound capable up to Dolby Digital 7.1, the PS3 is also able to apply a variety of advanced DSP filters in real time.

\section{The (re)positioning of subjects in the user experience}

The present section will explain how immersive realism has been constructed in the present sample of games, from the SNES to the Sony PS3. The foundational question at hand concerns how these games have been designed and what is encoded the design of their auditory experience. In the design of a game, a player is presumed 
— a game, after all, must be played — and this subject is positioned differently via the affordances of the system and game. It is especially important to keep in mind that like other media, all sounds present are there for particular reasons, with particular functionality. Given the limited resources levied to sound design and reproduction in game design, this isn't surprising, but moreover, as in other media, stimuli are included in order to position the subject in a particular way. This player-subject is positioned in the virtual world and game apparatus in learning to play the game, and in learning to play, there is a tacit acceptance (and a necessary acceptance) of the rules and mechanics of the game, and this includes the sensory regime employed, from visual perspective to auditory perspective. The game and system, as an apparatus, position the player in the illusion of the first-person perspective, visual but, as this entire study has sought to demonstrate, audile as well. To use the popular film theory term, the question at hand is how does the apparatus of the game "suture" the player to the virtual world (Silverman, 1983). The analyses of how each game individually positions its player-subject can be found in Appendix 2. This section will take these descriptions into account and explore how subjects have been (re)positioned diachronically, via the mechanics of the present games, and how these mechanics espouse particular regimes of how the game world is to be perceived, via their implementation of particular aesthetics, influenced by the aesthetics of realism in war cinema. The section will conclude by returning to the platform history and the questions of immersion and realism.

\section{Subject positioning over time}


Diachronically, across these four games, there has occurred a dramatic (re)positioning of the subject. Weaving the threads of technological innovation, the aesthetics of immersive realism, and subject positioning together, it can be seen that, across these four games, a shift has occurred in the aural gameplay experiences promoted by the apparatus of the console and game. To return to the Platform History section, and Collins (2008) in particular, four specific technological developments have had major impact on game audio: (1) newfound possibilities for 3D sound, (2) the introduction and refinement of real-time DSP and synthesis, (3) an increasing number of possible simultaneous samples, and (4) increasing bit depth and sample rates of digital audio samples as well as the increasing availability of system and game resources. Each of these will be explored in turn, in relation to the four games studied. Finally, this section will conclude by exploring the global effects of these four developments on the aural positioning of gamer subjects, returning specifically to questions of immersive realism and how it is promoted in the design of game audio.

\section{$\underline{3 D \text { audio }}$}

As has been noted, the introduction of 3D audio made it possible to emulate real-world hearing which, unlike vision, gathers stimuli in a 360-degree sphere. 3D audio is notably lacking from both Wolf $3 D$ and $G E 007$, although in the latter, the technology was available at the time. Halo: $C E$ features Dolby surround sound, but it uses the original stereo audio files as its source and is thus not true surround sound. $B F 3$, on the other hand, features true Dolby 5.1 surround sound (and the Sony PS3 supports up to Dolby 8.1) and, for those without a surround sound system, features rather convincing simulated surround sound. For the player-subject, this affords, and 
in many cases necessitates, a new modality of play. Whereas Wolf $3 D$ and GE 007 feature only mono or stereo sound, respectively (though GE 007 does not take advantage of any sort of positional audio in stereo space), $B F 3$ and Halo: $C E$ both feature 3D audio. Sounds are entirely non-localized in the former; regardless of the virtual location of their source, they all emanate from the same location. Navigation is a purely visual affair in such a space; movement is a guessing game where the player has little to no information regarding what will be around the corner, or what is coming up behind them. In the latter however, sounds are localized in 3D space. The player is afforded access to game action outside their field of vision and in a realistic way. Whereas prior attempts had used on-screen text or alternate camera views, 3D sound is unobtrusive. Navigation becomes just as much of a sonic affair as it is a visual one, but moreover, just as Chion (1994) finds the rendering of reality in film sound as necessary to compensate for the other senses that cinema is lacking, 3D FPS audio comes to fill in for the noticeable lack of true empirically 3D graphics in FPS games. This point bears repeating — while visuals in contemporary FPS console games are often described as 3D, they are merely a $2 \mathrm{D}$ rendering of real-time playercontrolled perspectival changes. 3D audio, such as that implemented in Halo: $C E$ and $B F 3$, comes to compensate for the dimensional limitations of the FPS visual perspective; it adds depth, fore and aft, and height and width beyond the frame of the television screen. For a fast-paced, point-driven game like online multiplayer in $B F 3$, this is crucial towards staying alive and earning points. Thus, while 3D sound has obvious implications for sensory immersion in its ability to inculcate a sense of presence in the player, in its functionality, it has far-reaching implications for 
challenge-based immersion as well, as can be seen in BF3. 3D sound, the information it provides to the player, inspires an anticipatory mode of gameplay in the FPS, where listening becomes a necessary activity. Saether (2011) has made this clear when he advocates the use of headphones for competitive play: "The use of headphones makes it especially easy to localize sounds, so that you know where on the map you can find their sources. It will be easier to play tactically, if you use sound as a factor while making your decisions in play" (para. 20). Coupled with the affordances of real-time DSP, 3D audio takes on an even greater role.

\section{$\underline{\text { Real-time DSP }}$}

Used often to simulate real-world acoustic phenomena such as reverberation and echo, real-time DSP augments 3D sound both in its realistic acoustic simulation and in the added possibilities for ludic functionality via the increased information realtime DSP provides the player. As the SNES does not afford it, Wolf $3 D$ is lacking any sort of real-time DSP. Wolf 3D's audio is stripped bare; it sounds cold and hollow digital. GE 007 does feature some real-time DSP, although rather simplistically as compared to its modern-day counterparts. However, by introducing proximitydependent amplitude for NPC sounds, the game allows for some audio-assisted navigation, especially important when there is no positional audio in use. The player can gauge their range from enemies and thus prepare for impending danger. Halo: $C E$ opened up new worlds for real-time DSP, going beyond just proximity-dependent amplitude. While, like GE 007, the game does not feature any real-time acoustic phenomena, it does utilize real-time DSP to break the monotony of repetitive samples, like gunfire, and in addition real-time DSP provides the foundation on which the 
game's overarching ambient ground is built — a very important development for imaginative immersion that will be discussed further in the next section. Essentially, real-time DSP provide dynamics and variability to game audio. In the case of Halo: $C E$, the use of real-time DSP adds a richness to gun sounds that is not found in previous games. Rather than a static sample of gunfire, the game permutates a variety of samples, from gunfire, to dropping shells, to incidental clicks and whirrs, randomizing them in novel ways. $B F 3$ continues this but, with a much more capable system and game engine, incorporate real-world acoustics. Continuing with the example of gunfire, not only is its amplitude proximity dependent, not only does it consist of much more than the same sample repeated until the player releases the trigger, but it occurs in a space that interacts acoustically with it, and all in a rich environment of 3D sound. The player is positioned in a virtual world of aural stimuli, not purely superficial as in Wolf $3 D$, but immensely functional, and thus has positive implications for challenge-based immersion. Rather than merely present, audio in BF3 provides important information to the player, and to the point that its absence can have a negative effect on a player's ability to perform in the game. Underlying all of this variability however is the third audio development, the possibility for many simultaneous samples.

\section{Soundscape density}

Undergirding the possibilities for an immersive audio experience via the affordances of 3D sound and real-time DSP is the possibility of many simultaneous samples. Richness and variability are not possible without many concurrent sounds and, moreover, the ability to design a sonic ground, as exists in Halo: $C E$ and BF3 is 
predicated on the possibility of a dense, yet functional, soundscape. The affordances of 3D sound and real-time DSP have been discussed at length; this last point, however, has not. As has been mentioned, Truax (2001) has understood a soundscape via a figure/ground relationship. In a very common urban environment example, certain sounds may be regarded as constants; even though the din of traffic is dynamic, or the honking of horns, or the idle chatter of passerby are dynamic, they are constants and provide a sort of sonic backdrop upon which other sounds are heard. The nearby clatter of a construction site blends into the background, but a passing siren likely does not; it is specifically designed to break the monotony and provide information to the listener, a figure sound. Importantly, for Truax, the listener is not a passive recipient of sound; rather they are an active participant in the construction of the soundscape. A passing listener on a city street projects their own sounds into the mix, from the rustling of their shopping bags, to the incessant pinging of notifications on their smartphone, all of which either get lost in the ground or emerge as figures, depending partially on who is listening and what for. Whatever the case, silence is rare, if existent at all as the legend of John Cage in the anechoic chamber goes (Cage, 1967, p. 134). Returning then to the soundscapes of FPS video games, a game without a sonic ground is lacking an important referent to the real world, but especially the world of sound in war cinema, which often features lush soundscapes and emotive scores. While this ground may not be entirely functional — though in many cases, such as where the ground is indicative of a certain key place or evokes tension in a moment of impending danger, it can promote challenge-based immersion — it can serve the 
purpose of drawing the player in, inspiring presence and promoting an immersive gameplay experience, largely at sensory and imaginative levels.

\section{System and game resources}

Without complete access to game audio assets, exploring system and game resources as they relate to audio reproduction in these specific games is limited. It cannot be known exactly which sounds are synthesized and which are samples, and moreover what the exact sample rates or bit depths are or how these vary throughout the game, based on soundscape density. Nor can it be gathered how exactly platform resources, such as processor speeds and available storage space limit audio reproduction in the game. However, some general comments should suffice. The resources afforded by the system and whatever storage media the game is distributed on, from processor speed to sampling rate to storage space, dictate the limits of the game's audio design. Thus, quite simply, as the technology behind game consoles and storage media has improved, so have the affordances of the game audio, including the possibility to reproduce sound in a manner that emulates reality, with high sample rates and bit depth, modified via real-time DSP and positioned in 3D space.

\section{$\underline{\text { Subject positioning and immersion }}$}

Ultimately, when these four developments are coupled, the possibilities for an immersive gameplay experience are heightened. Designing an experience immersive at all three of Ermi and Mäyrä’s (2005) levels (sensory, challenge-based, and imaginative) becomes possible via sound. Sensory immersion can be accomplished via 3D sound, challenge-based immersion via the conflation of 3D sound and real-time DSP, and imaginative immersion via the use of dynamic bodily sounds or the 
introduction of a sonic ground, used to instill a mood or sense of tension/relief. Tightly intertwined with these three types of immersion is the representation of realism. The aesthetics of realism represented via these technologies has been informed by codes of realism, to use the language of Chion (1994), discursively constructed via radio, drama, film, and television, not only in the content of FPS audio, such as the sounds of automatic gunfire, the staccato profanity-filled dialogue, or the roar of a nearby jet engine, but in how this content is presented. 3D audio and real-time DSP, and the acoustic effects they generate, are not neutral technologies; they too adhere to particular aesthetics of realism which can be found in war cinema. One particular example makes this very clear: hearing loss is the most prevalent disability veterans face (Veterans Benefits Administration, 2011), yet viewers of war cinema rarely see combatants writing on the floor in pain after shooting a machine gun indoors. Similarly, FPS gamers do not often experience prolonged virtual hearing loss during gaming sessions. Though some games, like $B F 3$, simulate tinnitus after a nearby explosion, the effect is short lived.

These various technological developments, as has been demonstrated, came about on a trajectory towards increasing the immersive realism of designed virtual worlds, and in adherence with the aesthetics of realism as presented in war cinema. Both sensory and imaginative immersion support this and can be found in the experience of cinema. The prospect of challenge-based immersion, however, is not found in cinema. As has been discussed at length in this study, a defining trait of video games is that they are interactive; the prospect of challenge-based immersion is a result of this. While the various non-diegetic sounds, such as interface and pickup 
sounds, coded in this study may metaphorically relate in representation to the events of the game (such as the file and paper sounds in the GE 007 menu), they bear little resemblance to the aesthetics of realism as found in war cinema. This makes complete sense as there is no reason these sounds would even be found in cinema; they are a result of the interactivity in video games. A traditional film does not have health or ammo pickups, nor does it feature menus or other interfaces, and thus does not require sounds for these things. Thus, if FPS video games utilize the aesthetics of realism as present in war cinema, they do so only insofar as they enhance the possibilities for immersive realism in the game. Yet, they go beyond these aesthetics in the pursuit of ludic functionality. A video game is a text that can be analyzed as a narrative, as a system of symbols, but it is also a system of rules. The peculiarity of immersion in the FPS, that which sets it apart from immersive experiences that may occur in other media, is that it occurs at the intersection of both of these points. What is advanced about a game like $B F 3$ over Wolf $3 D$ is not merely that it sounds more "real," or at least in accordance with the aesthetics of realism from war cinema — though certainly it does - but moreover that it unifies realistic representation with the interactivity of simulation. This interactivity is enhanced via a symbolic system of noises - some abstract, some metaphorical — that provide feedback to the player about their interactions in the virtual world. The result is a unified stream of audio information, simultaneously and seamlessly narratological and ludological — representative and simulative.

Merging these threads, the player-subject of FPS games has been aurally (re)positioned on the same technological trajectory, sutured to the virtual world via the 
mechanics and aesthetics of immersive realism and their shifting affordances and disaffordances. Technological development and the aesthetics of realism, as well as the evolution of FPS games into competitive multiplayer formats, have been mutually influential in this, one necessitating the other in a constant cycle of refinement and occasional decline. These are the material conditions in which the player is made a subject in, and subject to, the audible world of the FPS. 


\section{CHAPTER 5}

\section{CONCLUSION}

\section{Summary of findings}

This study has explored the following research questions:

$R Q_{1}$ : Has the pursuit of immersive realism in FPS gaming led to the emergence of new technologies related to audio reproduction? If so, why, how, by whom, for whom, and through what means have these technologies emerged?

$R Q_{2}$ : As audio reproduction technologies develop, how are they implemented in FPS sound design?

$R Q_{3}$ : How do the changing affordances of FPS games, as brought about through the implementation of new audio reproduction technologies in the sound design process, (re)structure the possibilities for auditory experience?

Overall, this inquiry has generated significant responses to these questions. For $R Q_{1}$, it has been demonstrated through an examination of various marketing materials and industry interviews that the pursuit of immersive realism in video game play has brought about changes in audio reproduction technologies. The context of these developments, especially as they relate to the aesthetics of realism and their discursive construction have been explored at length. For $R Q_{2}$, this study embarked on a platform history of game consoles, beginning with the pre-history of gaming as we know it today, attempting to bridge a gap between historical and scientific/technological inquiry, by concurrently examining both how and why these technologies are 
implemented. For $R Q_{3}$, this study took a sample of four first-person shooter (FPS) games and coded them based on how they positioned the listening player-subject within the apparatus of the game and console. These results were then brought to bear on the platform history in order to offer a diachronic account of how console FPS games have (re)positioned their subjects alongside the development of new technologies related to audio reproduction. Ultimately, these findings demonstrate a mutual influence between technological development, the aesthetics of realism, and the positioning of subjects as gamers - as interactants and not mere inhabitants. While this study has provided novel insight at the intersection of sound studies and game studies, it is not without limitations and avenues for further research.

\section{Limitations and further research}

In this study's attempt at breadth, it is lacking in exhaustiveness at points. Two particular areas could greatly benefit from deeper research. First, in demonstrating how technologies have been developed in the pursuit of immersive realism and, furthermore, how the aesthetics of immersive realism have been constructed discursively, this study is lacking deep archival research. Archival research at various game, film, television, and radio repositories could greatly benefit the present research and add a layer of exhaustiveness that could truly account for the material "why, how, by whom, for whom, and through what means" questions originally proposed by Sterne (2003) in his study of the transducer. While finding marketing materials and developer interviews relating to recent games like Halo: Combat Evolved and Battlefield 3, as well as their respective consoles, did not present much of a challenge, finding the same for Wolfenstein $3 D$ and GoldenEye 007 proved quite difficult. Part of 
a much larger issue pertaining to the preservation of popular culture, cultural products such as magazines and commercials relating to these games and their consoles are difficult to come by, if still existent at all.

Coupled with archival research, deeper technical analysis could greatly benefit this study, such as the kind found in various "platform studies" works like Montfort and Bogost (2009) and Monfort et. al (2013) that explore deeply the underlying computing processes that provide the affordances and disaffordances of the game and console. It is these underlying processes that most fundamentally dictate what the apparatus can and cannot do. Deeper, and much more technical inquiry here, might allow for a more nuanced discussion of gaming, sound, and subject positioning in a way that accounts for the immense amount of complexity involved in reproducing sound in digital media.

Finally, the last avenue for further research is not as much a limitation of the present study as it is reliant on it. Fundamentally, this study has been concerned with the aesthetics of realism have been utilized in FPS video games to construct immersive gameplay experiences via audio. This study has not been concerned with players themselves and has not been guided by the format of audience analysis and the data from player self-report. Rather, its concerns have been with game texts and how they structure the user experience. Before it can be known whether or not a game is sonically immersive, "immersion" and how experiences deemed immersive are constructed, must first be defined, an important part of this present study. Thus, an important next step would be audience analysis, actually examining whether or not the demonstrated aesthetics of immersive realism truly are effective towards immersing 
the game's player-subject and, furthermore, how the technological advances demonstrated in this study alter the way, if they do at all, players have approached FPS games.

\section{Gaming as a cultural modality}

Without rehashing the various statistics expressed earlier in the study, gaming has become a ubiquitous cultural modality, and increasingly so. While this study has examined console gaming, video games have moved beyond the necessity of owning a gaming-specific device. Smartphone users occupy a significant portion of the gaming market, with 56\% percent of U.S. adults owning smartphones (Smith, 2013), 64\% using their phones for gaming, and gaming occupying almost $25 \%$ of the average smartphone user's app consumption (GO-Gulf, 2012). Furthermore, to return to the console, even those systems once dedicated to gaming have transformed into allpurpose media centers. Looking at this year's new generation of consoles, the Sony Playstation 4 and the Microsoft Xbox One, the rhetoric is telling. Microsoft heralds the Xbox One as ushering in a "new generation of games and entertainment" (Microsoft, n.d.). The Sony Playstation 4 similarly frames their system's capabilities as an entertainment device, from its incorporation of a Blu-Ray drive, to the various apps available in the Sony Playstation Store, such as Netflix and Amazon Instant (Sony, n.d.). It is worth noting that these consoles are still marketed first and foremost as gaming consoles, but in their inclusion of other entertainment possibilities (and both of their console predecessors, the Sony Playstation 3 and Microsoft Xbox 360 offered similar capabilities) they have signaled a shift in gaming from a niche activity to a 
universal one. As Bogost (2011) notes, "videogames are [no longer] a subcultural form meant for adolescents but just another medium woven into everyday life" (p. 7). Similarly signaling the growth of gaming as a major cultural modality is the at-present widely applied practice of gamification. Gamification entails the application of game mechanics to contexts outside of gaming, such as the earning of points for completing desired tasks. From pop-up ads to customer loyalty programs, gamification has been used extensively for marketing purposes. But moreover, it has found uses in education, workflow management, social media, workplace training, and even the practices of modern warfare. In light of the present study, this last one deserves further attention. In The Gulf War Did Not Take Place, Jean Baudrillard (1995) maintained that, while the events spearheaded by the United States against Iraq in 1990-1991 actually did take place, their constant and stylized (mis)representation in the media, confused reality and narrative/simulacra, making the two impossible to separate. The actual events became obscured and replaced by the hyperreality depicted by the media. While Baudrillard was not talking about video games, his point is relevant. Through the processes of gamification in warfare, reality is replaced with hyperreality, and likely with tremendous moral implications. Two particular examples should help to clarify this point.

As the summer of 2013 concluded, the National Security Agency (NSA) of the United States became wrapped up in a fresh scandal surrounding their "spying" activities of U.S. citizens as well as citizens and politicians abroad, broken by former analyst Edward Snowden. Without rehashing the entirety of the scandal, in August of 2013 Germany's Der Spiegel (Poitras, Rosenbach, \& Stark, 2013) broke a story 
detailing the NSA's data-gathering activities upon German citizens, and without total transparency to German government. Tied in with this scandal was the NSA's controversial Xkeyscore software, first revealed by Snowden in July 2013. Essentially, XKeyscore, used by low-level NSA analysts, has greatly expedited the processes of monitoring phone calls, emails, browser histories, and various other communications, all undertaken from a central location and without need for approval. As the report from Der Spiegel notes however, this is not mundane software like Microsoft Word, where analysts simply gather and log data. Rather, it has a particularly game-like system of incentives: "To create additional motivation, the NSA incorporated various features from computer games into the program. For instance, analysts who were especially good at using XKeyscore could acquire 'skilz' points and 'unlock achievements"' (para. 20). Tied up with another recent ongoing scandal, if not multiple, the use of drone strikes by the United States in accomplishing military strikes has come under wide criticism. Many being controlled domestically at Creech Air Force Base in Nevada, as a Bureau of Investigative Journalism report notes, up to 418 reported drone strikes have been undertaken since 2004 in Pakistan, Somalia, and Yemen, with over 120 in Pakistan in just 2010 (Woods, Serle, \& Ross, 2013). Mediated via joystick controls, a computer keyboard, and multiple screens, the experience, as one former drone operator notes, "is a lot like playing a video game" (Brown, 2013).

What do these two examples mean for gaming as a cultural modality? And what do these examples of the gamification of modern warfare have to do with the present study? While this paper has explored games as they exist as entertainment, 
what these examples demonstrate is that the modality of gameplay and its entwinement with simulation and realism has implications far beyond entertainment. The present study could just as easily be applied to an analysis of military vehicle simulators or the interfaces through which drone operators operate, examining how realism is represented and immersive experiences constructed via the technological affordances offered by the system, and how these systems attempt to promote immersion, both through realism and functionality or, perhaps in the case of drone operators, distance and separation. While this study has refrained from discussing the political and moral implications, nevermind psychological, of FPS representations, instead offering FPS games as the best case study for an examination of immersive realism, certainly these implications exist and are many. But no less deserving of a critical attitude are the technologies through which games are mediated and the other forms these technologies take, whether that be the interface between a drone operator and their target or the earning of points on a Starbucks loyalty card. Criticality at the level of gaming as popular culture is an important precursor to criticality at various levels of gamification, from the mundane to the violent.

Where gaming is going is anyone's guess. But with the release of the Playstation 4 and the Xbox One, it can be sure that the goals of immersive realism are still guiding the development of game technology, not the least of which concern audio reproduction. Both systems promise increased immersive realism, as compared with their predecessors. Sony promises, via their systems advanced technological capabilities, "new games with rich, high-fidelity graphics and deeply immersive experiences" (Sony, n.d.) while Microsoft promises to "push the boundaries of 
realism" (Microsoft, n.d.). Moreover, starting where the early 90s left off, the prospects of virtual reality have once again found their way into game development. The Oculus Rift, currently in development, is a VR headset that promises to "change everything," offering immersive gameplay experiences like never before, and in a budget-friendly format (Oculus VR, n.d.). Whatever the next decade may bring for technological developments in gaming, it can be sure that the pursuit of immersive realism is not going away anytime soon.

\section{$\underline{\text { Conclusion }}$}

This study has been my attempt at answering the call put forth by Lowood (2006), speaking at the merger of studies in the history of science and game studies:

[Game studies] potential lies in critical engagement with games as symptoms of and impacts on society and culture - contextualizationbut in ways that illuminate the structure of revolutions in design and game play. Such game studies will speak, eventually, to both academic scholars and enlightened developers; it may be possible to realize the goal of moving inside the black box along mutually traveled paths more successfully than historians of science have done in the eyes of many scientists. (p. 81)

By interrogating how immersive experiences have been constructed via FPS game audio and aesthetics, and the subject positioned in these experiences, diachronically, the "black box" becomes slightly more transparent. As video games and the modality of gaming proliferate as dominant cultural forms, this criticality becomes more important than ever. Video games are commodities, complete with particular marketing strategies, but video games are also one of the few places where subjects can enact a virtual world, can simulate action and activity once only possible in dreams, and, if just for an hour, be somebody or something else. Other media may offer similar possibilities, but they do so in differing ways. Game, unlike 
representational media like films and literature, are meant to be played; they are interactive and engaged with in differing ways — via simulation. There are obvious moral implications here and certainly simulation can be undertaken with nefarious aims, but video games and the modality of gaming hold promise — the promise of endless creativity. A critical mode of engagement need not curtail an enjoyable mode of engagement. While watchful eyes (and ears) should always remain critically fixed on media, especially as they transform and proliferate from lesser status to dominance, the hands need not, and should not, remain idle. Games must be played to be understood, to be praised, or to be contested. Games should not only be witnessed in their graphical elements, but listened to, as aesthetic constructions and as actionable sonic worlds, that serve to position their players in particular sensory regimes, emergent from particular historical and cultural contexts, and implemented via the conflation of particular technologies. 


\section{APPENDICES}

\section{Appendix 1 - Textual analysis procedures}

\section{Typology construction and subject positioning}

A variety of typologies for describing game audio have been forwarded. The present coding scheme represents a combination of these, culling the superfluous and redundant and emphasizing where they overlap. The literature has been broken down into two categories, those which seek to describe game audio elements from the perspective of game production, development, and design, accounting for the types of game audio assets and how they are organized in the development of the game (Production based), and those which seek to describe these elements from the perspective of the player, interrogating what they mean and what they do for the subject in gameplay (Meaning based). I will first briefly review the literature and then proceed on to explain the typology employed in the present study.

\section{$\underline{\text { Production-based }}$}

Folmann (2004) has distinguished four categories of game sound: "vocalization," which includes both avatar vocalizations and external communications like VOIP; "sound-FX," both in object interactions and interface sounds; environmental/ambient fx," concerning the production in a development setting of the ambient soundscape backdrop; and "music." As a game audio composer, it is perhaps no surprise that his approach is from the perspective of audio implementation at the stage of game development. Similarly, Friberg and Gärdenfors (2004), both designers working with games and interactivity for the blind, have distinguished five categories 
of "audio information" present in video games: "avatar sounds," "object sounds," "character sounds," "ornamental sounds," and "instructions" (p. 4). Missing from both typologies are the means to describe a player's real-time interaction with a game through sound. Both Folmann (2004) and Friberg and Gärdenfors (2004), were concerned with constructing typologies relevant at the stage of development - with what sounds are and the types of information they can offer a player — and not the stage of gameplay, which should reflect a concern not only for what sounds are, but for what they do, dynamically, for the player and how, situated in actual practice. The variety of sound objects present in the auditory world of a game are systematized adequately (though perhaps not exhaustively) but neither Folmann (2004) nor Friberg and Gärdenfors (2004) have systematized a way in which to describe how game sounds positions the player as a subject in a virtual world through the promotion of an immersive gameplay experience. This is not to demean their work in any way, as describing game audio from this perspective is seemingly not their goal; it is merely to point to their work's limitations towards the present study.

\section{Meaning-based}

Stockburger (2003), in one of the earliest forays into systematizing game audio, has augmented a typology of "sound objects" with a description of auditory position through the diegetic/non-diegetic framework most commonly associated with film sound. In doing so, he not only has described game audio in terms of what it is or what it signifies, but also its context of interpretation in the game world in gameplay, an important factor when seeking to describe player immersion. Stockburger's sound objects consist of three which occur largely in the diegetic part of the game 
environment: "speech"; "effect," which includes sounds which are connected to objects or events in the game environment; and "zone," which are linked with game locations. The last two, "score" and "interface" are not part of the diegetic environment. Furthermore, Stockburger has examined two "spatialising functions" particular to video games, stemming largely from the work of film theorist Michel Chion. The first concerns acousmatic sound; the second concerns the "spatial signature" of a sound, a concept of particular important that will be returned to.

Collins (2008) has conflated auditory position with interactivity in her description based on the participatory and non-linear aspects of game sound. Like Stockburger, she has distinguished between diegetic and non-diegetic sound (while noting that such a dichotomy may be ill-suited to the study of game audio). Within both diegetic and non-diegetic sound, Collins has distinguished between three degrees of interactivity: dynamic audio that is adaptive, dynamic audio that is interactive, and non-dynamic audio, resulting in six distinct types of game audio. Her goal has not been necessarily to describe the types of sounds utilized in developing a game, like Folmann (2004) and Friberg and Gärdenfors (2004), but rather to offer a manner of deconstructing the dynamics of game audio as they function in real time and, moreover, deconstructing how the dynamic soundscape of a game positions the subject.

Much of the work featuring Mark Grimshaw at the helm (Grimshaw, 2007a, 2007b; Grimshaw and Schott, 2007; Grimshaw, Charlton, and Jagger, 2011) similarly has explored the positioning of audio in the diegesis, interactivity, and the functions of game audio in order to better understand player immersion. Diegetic sounds are 
broken down to include sounds that are "ideodiegetic," which include the immediate sounds a player hears, and sounds that are "telediegetic," including sounds produced by other players that affect the present player. Furthermore, like Stockburger, a focus was levied to sound as it is utilized in gameworld navigation. Utilizing the language and theory of Schafer (1977), Grimshaw et al. have explored how game audio can function as an "auditory beacon" by anchoring the player in space, how certain sounds can be located as the "keynote sounds" of a game location, and how certain sounds function as "sound signals," standing out from the sonic ground, to echo Truax (2001), and providing information relevant to game action.

Van Tol and Huiberts (2008) in their "IEZA" (interface/effect/zone/affect) model have presented a multi-dimensional framework for understanding game audio. Preserving, again, the diegetic/non-diegetic dichotomy, they distinguish two types of diegetic sounds and two types of non-diegetic sounds. Furthermore, within this breakdown they have distinguished between two dimensions of information: sounds that convey information about the activity of a game and sounds that convey information about the setting of a game. On the diegetic half of the model, Van Tol and Huiberts have distinguished between "zone" sounds, which correspond to the virtual environment in which the game is played, and "effect" sounds, which correspond with specific sound sources in this game environment. Zone sounds thus convey information about the setting of the game and effect sounds convey information about the activity. On the non-diegetic half of the model, they have distinguished between "affect" sounds which express the setting of the game through 
non-diegetic means, such as music, and "interface" sounds which provide information about player activity through non- diegetic alert sounds.

While the literature reviewed above offers a lot to the study of game audio, all are limited in some regard. The production typologies discussed do not account for player interaction and, out those discussed that do account for player interaction, none comprehensively account for the implementation of audio reproduction technologies like positional audio and real-time digital signal processing (DSP). However, by merging together these disparate sources, a typology has been created more adequate to the study at hand, one that accounts not only for the various types of sounds and their origins, but also where they are positioned in the diegesis, what functions they can serve for the player, and how they are processed by the game engine in a manner that promotes an immersive gameplay experience.

\section{Typology of FPS audio}

The following description details the typology shown in Appendix 1.

\section{$\underline{Y \text {-axis }}$}

Examples: What is the sound object in specific? Where is the sound being heard? Who/what is the source? What does it sound like?

Positional audio: Is the sound located in stereo space? Is three-dimensional (3D) audio (surround sound or simulated surround sound) being implemented in some way? Does the sound function as an "audio beacon," helping to anchor the player in space (Grimshaw \& Schott, 2007)?

Real-time DSP: Is real-time DSP being used on the sound object in a noticeable way? How? What is the context? Does the sound have a "spatial signature," i.e., is it 
marked by real-time reverb or echo to instill a sense of real-world acoustics (Stockburger, 2003)?

Diegetic positioning: This study has relied on Grimshaw and Schott's (2007) typology of diegetic positions. Diegetic sounds are broken up into Ideodiegetic, the immediate sounds a player hears, present in the diegesis, and Telediegetic, sounds produced by other players that affect the present player. All other sounds are considered Non-diegetic.

Semiotic distinctions: What sort of information is being conveyed? Is there an aural distinction as this information changes? E.g., does the sound of a weapon change as it runs out of ammo? Do bodily sounds change as health is depleted? Is the score "triggered," indicating an impending change in the game state?

Figure/ground: This category concerns the theory and practice emanating from the field of acoustic ecology, with specific attention to the work of Truax (2001). How does the sound fit into the soundscape? Does it act as a keynote, providing a steady aural backdrop that could be used to identify the space? Does it function as a sound signal or aural figure, standing out from the ambiance of the space?

\section{$\underline{X-a x i s}$}

Referential sound objects: The X-axis consists of the variety of contextual sound effects present in the game. The following categories are coded for sounds that are produced in actions by the player and for sounds that are produced in actions by non-player characters (NPCs). These categories were chosen based on the literature of Droumeva (2011), Stockburger (2003), Folmann (2004), 
Van Tol (2008), and Friberg and Gärdenfors (2004), but altered slightly to directly relate to FPS games. Sounds are broken down into: Vehicle sounds, Object sounds, Movement sounds, and Body sounds.

Other sound objects: The following sound objects are not strictly referential. These categories were chosen based on the literature of Droumeva (2011), Stockburger (2003), Folmann (2004), Van Tol (2008), Grimshaw and Schott (2007), and Friberg and Gärdenfors (2004), albeit with slight alterations to relate more to FPS games. Sounds are broken down into: Speech/dialog, Environmental sounds, Interface sounds, Alert sounds, and Music.

\section{$\underline{\text { Data collection }}$}

Games were played in sessions no longer than two hours, to avoid mental fatigue. First-player campaigns were played through fully in each game, and multiplayer modes were tested at length, until subject positioning could be satisfactorily described. A few principles, geared towards evidencing sonic diversity, guided the process: (1) Environmental variation — Differing game environments (e.g., wide open fields, empty concrete storehouses, small rooms) were sought in order to best showcase any 3D audio and real-time DSP geared towards avatar navigation in the virtual world; (2) Weapon/vehicle variation - Different weapons and vehicles employed in the virtual world to evidence any variation and again to showcase how real-time DSP, if any, altered different weapon sounds in different spaces or due to different aspects of physical simulation (e.g., disconnect between hearing and seeing rocket-propelled grenade explosion in BF3); and (3) Game mode variation Different game modes, such as single-player campaign modes and multiplayer modes 
(both online and in-person, if available) were tested in order to showcase any design differences in more hardware-intensive game variations and, in addition, how the introduction of human-controlled non-player characters altered the sound design, either via the inclusion of new elements, the alteration of elements existing in singleplayer modes, or the exclusion of previously present elements.

\section{$\underline{\text { Sample selection }}$}

\section{$\underline{\text { Wolfenstein } 3 D}$}

Released on May 5, 1993 for the SNES, Wolfenstein 3D (Wolf 3D) marks the emergence of the FPS for the console platform. Developed by id Software and published by Apogee Software, the game was originally released in 1992 for the MSDOS PC platform, but has since been ported to a variety of other computer and home console platforms. Its legacy is hard to overstate: Wolf $3 D$ can claim a good deal of the responsibility for the run-and-gun archetype that has come to define the FPS.

Unfortunately, historic reviews are hard to come by; Nintendo Power, however, gave it a combined average of 3.75 out of 5 in 1994 (p. 107). Perhaps more telling are the amount of "best of" lists the game, and its various ports, finds a place on (IGN, n.d.; GF, n.d.; Mackey, n.d. — which are just a small sample) and the various awards and accolades that game has been awarded, including a CODiE award for "Best Action/Arcade Program" (CoDIE, n.d.) and a Shareware Industry award for "Best Action/Arcade Game (SIA, n.d.). The August 1994 issue of PC Gamer even called the game "one of the eight most important games ever released" (as cited in Apogee Software, 1995). Complete sales data is unfortunately unavailable for both the SNES port and the original MS-DOS version. 


\section{GoldenEye 007}

GoldenEye 007 (GE 007) was released for the Nintendo 64 (N64) game console on August 23, 1997. It is the 17th best-selling game of the 1990s, and the 1st best-selling FPS (Mazel, 2009), and to date has sold 8.09 million units, globally (VGChartz, n.d.). It has "universal acclaim" on Metacritic.com, with a metascore of 96, based on 21 critics, and a 9.2 based on 194 player ratings (Metacritic, n.d.). Gamespot.com, upon release, gave GE 0079.8 out of 10 compares the game's staying power with that of Mario 64 (Gerstmann, 1997). IGN.com awarded the game a 9.7 and the IGN community has awarded the game a 9.4, based on 5,939 player ratings (Perry, 1997). 1UP.com considers it one of the 60 most influential games of all time that "paved the way for the later popularity of Halo, Call of Duty, and more" (1UP, n.d.). As Markham (2012) notes, its multiplayer mode provided the archetype for future FPSs (Markham, 2012). But this was only one of GE 007's many innovations. It pioneered the "headshot" and FPS sniping with an adjustable scope and context-aware enemy reactions. Its requirement for objectives to be completed before the mission could be finished overhauled the FPS, normally solely focused on killing NPCs and navigating to the end of the level in one piece, transforming the "completionist" player to the "explorer" player. Its stealth mechanics and realistic employment of particular weapons for particular scenarios moved away from the typical "bigger and louder is better" mentality of many prior FPS games. While its graphics and sound design may appear crude from today's standards, its player and level mechanics truly revolutionized the genre.

\section{Halo: Combat Evolved}


Halo: Combat Evolved (Halo: $C E$ ) was released on November 14, 2001 by Bungie Studios as a launch title for the Xbox console system. Since its release, it has sold 6.43 million units globally (VGChartz, n.d.). By April 8, 2002, the game had sold one million units - setting the record for the fastest million-unit seller of any nextgeneration console (Microsoft, 2002). The first installment in the best-selling franchise, Halo: $C E$ has earned a 97 on Metacritic, based on 68 critic reviews, and an 8.5 based on 712 player ratings (Metacritic, n.d.). Gamespot.com awarded the game a 9.7 out of 10, calling it "one of the best shooters ever, on any platform" (Fielder, 2001). IGN.com also awarded the game a 9.7, with a 9 for graphics, and 10 for sound, and a 9 for lasting appeal (Boulding, 2001). With its two weapon player limit; trinity of weapon, melee, and grenades; multiplayer co-operative player; superb sound and graphics; and modding abilities, Halo: $C E$ brought some of the best aspects of FPS for PCs to the console and, while it being the greatest FPS of all time is debatable, is certainly one of the most influential.

\section{$\underline{\text { Battlefield } 3}$}

BF3 was released on October 25, 2011 for the PS3, Xbox 360, and the PC platform. The decision has been made to focus on the PS3 version for this study in order to best showcase the differences between consoles (Halo: $C E$ is Xbox exclusive). As of July 6, 2013, the PS3 version has sold 6.5 million units (VGChartz, n.d.). Cross-platform, the game has sold 12.88 million units, not including digital downloads or other means of acquisition, making $B F 3$ the 6th of the top ten selling FPS games of all time (D’Angelo, 2012). The game has earned a metascore of 85 on Metacritic.com, based on 38 critic reviews, and a user score of 7.4, based on 1,526 
reviews (Metacritic, n.d.). Gamespot.com awarded BF3 an 8.5, considering its multiplayer mode "among the best in its class, providing immensely rich and immersive combat zones" (Watters, 2011). IGN.com awarded the game a 9.0, while the user community on the web site awarded it an 8.9 , based on 2,553 ratings, both citing the multiplayer as the best in its class, alongside the sound and lasting appeal (Eykemans, 2011). In addition, the game has received multiple awards, including the 2011 IGN People's Choice Awards for "Best Shooter" and "Best Multiplayer Game" as well as a variety of "Best of Sound Design Awards" (G4, 2012; The Game Effect, 2012; Gamespot, 2012).

\section{Playing rigs}

\section{$\underline{\text { Wolfenstein } 3 D}$}

Due to difficulty acquiring an original SNES, Wolf $3 D$ was emulated on a Windows 8 computer using ZSNES v. 1.51 emulation software. Sound was sampled at $44.1 \mathrm{kHz}$ and routed via the emulator through the onboard sound card (Realtek ALC269 digital-to-analog converter) and to a Topping TP-20 power amp connected to two Polk Monitor 30 passive speakers for two-channel sound. Headphone listening used a pair of JVC HARX700 studio headphones, powered by onboard amplification. Audio playback was kept as period authentic as possible.

\section{GoldenEye 007}

An original N64 was used in coding GE 007. Audio was outputted via stereo RCA cables directly to a Topping TP-20 power amp connected to two Polk Monitor 30 passive speakers for two-channel sound. Headphone listening used a pair of JVC HARX700 studio headphones, powered by a Bravo Audio Class A tube preamp. 


\section{Halo: Combat Evolved}

Forwards-compatible, Halo: $C E$ was played on an Xbox 360 system, the console directly succeeding the original Xbox. For two-channel speaker playback, audio was routed from the console via stereo RCA cables to a Topping TP-20 power amp connected to two Polk Monitor 30 passive speakers. For headphone playback, the console was routed through a Bravo Audio Class A tube preamp and to a pair of JVC HARX700 studio headphones. Analog surround sound was routed from the console to a powered Logitech Z506 5.1 system.

\section{$\underline{\text { Battlefield } 3}$}

A PS3 "slim" edition was used in coding BF3. For two-channel speaker playback, audio was routed from the console via stereo RCA cables to a Topping TP20 power amp connected to two Polk Monitor 30 passive speakers. For headphone playback, the console was routed through a Bravo Audio Class A tube preamp and to a pair of JVC HARX700 studio headphones. Analog surround sound was routed from the console to a powered Logitech Z506 5.1 system.

\section{Appendix 2 - Subject positioning per game, description}

\section{Wolfenstein $3 D$}

Wolf $3 D$ was released for the SNES in 1993 by Imagineer, ported from the original home computer version released in 1992 and developed by id Software. Distributed as ROM cartridges, resources were quite limited in regards to sound design. Overall, the game's sound design is simplistic and representational, lacking the interactivity necessary for a proper simulative experience, at least as compared with 
contemporary console FPS games. However, it made the FPS a viable genre for the home console and provided the prototype for the format.

\section{$\underline{\text { Player sounds }}$}

As noted, sound in the game is simplistic, lacking variety, any sort of positioning in stereo space, or real-time DSP. In terms of variety, sounds created by the player are limited. Movement sounds are entirely non-existent. Bodily sounds are few, consisting largely of a couple similar "grunting" samples used to indicate when the player has been shot. There is more variety in object interaction sounds, including samples for each weapon in the game, but the samples do not vary, as either a real weapon or weapon sounds from cinema and television do; the exact same sample is repeated whenever the gun is fired. The game utilizes no positional audio nor any sort of real-time DSP, nor do any of the bodily sounds or weapons sounds offer any sort of semiotic distinction, i.e., when closer to death or when low on ammo. Synthesized gun samples offer little similarity to their real-life counterparts.

\section{$\underline{\text { Non-player sounds }}$}

NPC sounds are similar to player sounds in terms of limited variability and lacking resolution. Again, no positional audio or real-time DSP is present. Object sounds include enemy gunfire, which does sound different depending on the weapon used. The only present bodily sounds are grunting when shot and slightly different grunting when killed — one of two present semiotic distinctions. The second presents an audible difference in pitch between enemies of different difficulties, including pitchshifting down their gunfire as well as grunts. Just as with player sounds, no movement sounds are present. In terms of diegetic positioning, these sounds, as well 
as Player sounds, are all positioned ideodiegetically in the language of Grimshaw and Schott (2007), i.e., immediately heard by the player and present in the diegesis.

\section{Other sounds}

Speech and dialog in the game are limited to occasional pre-recorded "Halt!" commands from enemy NPCs and various growls and grunts. There is no environmental ambient sound of any sort and the only player-environment interactional sound present is the opening of doors, all of which utilize the same sample and offer no semiotic distinction between types of doors. Interface sounds include menu click sounds as well as a metaphorical cash register opening sound upon tallying awards points at the successful completion of the mission. These interface sounds are positioned non-diegetically. Alert sounds are few but include different, semiotically distinct sounds when picking up health restoratives, keys, treasures, and ammo. There is also an orchestral hit played when killed. These alert sounds are also positioned non-diegetically. Finally, the game's music consists of a looping MIDI score, composed and arranged by Bobby Prince, that varies for each level.

\section{Summary}

Wolf $3 D$ is certainly lacking aural realism in its use of low-resolution audio, at least as compared to the aesthetics of realism in war cinema. Truly, what may most promote an immersive gameplay experience, or at least historically so, is its novel employment of the first-person perspective. A designed identification with the eyes of the game's main character implies an identification with his ears. Promoting a sense of sensory immersion, the game positions the player visually as B.J. Blazkowicz, its main character. Aurally, the game does very little to identify the player with a first-person 
perspective, largely because the technology simply was not available at the time to do so, like real-time DSP and 3D aural positioning. Audio is presented as a centrally located point in a 180-degree field, a composite of isolated sounds all emanating from the exact same location. Yet, even without the possibilities of real-time processing and spatialization, the developers manage to find other, albeit less effective ways, of promoting an immersive experience, at all three of Ermi and Mäyrä's (2005) levels from the incorporation of player grunts when shot to the ability to trigger alert sounds when picking up various items. While the game may be lacking in faithful reproduction of the aesthetics of war cinema, from accurate depictions of weapon sounds to the inclusion of movement sounds, the game offers a sense of immersion in the ways in which it is interactive. In this way, Wolf $3 D$ resembles earlier video games where audiovisual realism was just not possible with the present technology; such games, like Tetris, to offer a common example, are still capable of offering immersive experiences, though perhaps not at the sensory level. Wolf $3 D$ follows this path with its sonic elements of ludic functionality, like health pickups, but in incorporating elements of audiovisual realism that mesh with war cinema, it opens up the door for new types of immersion and creates a need for new technologies of reproduction and representation that can provide these immersive experiences. Thus, where the game may most promote a sense of immersion through sound is in the player's acceptance of its limitations, of its "reduced" realism, to use the language of Grimshaw (2007a). While the audio, on its own, may not be able to provide a sense of presence in the virtual world, coupled with an acceptance of the first-person visual perspective and the ways in which sound provides actionable information to the player, it has a chance. In 
this, the game set the stage for the FPS as a viable genre. In spite of its shortcomings, the game's audio provided an early, barebones model of FPS audio.

\section{GoldenEye 007}

GE 007 was released for N64 in 1997 and has since been heralded as one of the most important FPS games ever. Agenda-setting in its use of authentic weapons (albeit renamed due to legal reasons), anti-“completionist” mode of play, control and navigational schemata, and multiplayer formats, the game, like Wolf $3 D$, set the stage

for much that would come after. While strikingly confined due to the limitations of the system and the resources of the ROM cartridge — in an interview with Designing Sound, Graeme Norgate, sound designer for the game, noted he only had 700k of available space in which to store the entirety of the game's audio (as cited in Designing Sound, 2010) — the game's audio is leagues beyond Wolf $3 D$. The game is vast for its time, with nineteen single-player levels, six multiplayer-only levels, over thirty usable weapons, a wide variety of usable gadgets, and a wide variety of characters. If audio sample rates and bit depth suffer because of this, it should be no surprise given the limitations of the cartridge and console. Yet, in spite of these limitations, GE 007's audio features wide diversity and various complex mechanics that serve to position the player in a soundscape far removed from that allowed by the SNES. The game does not offer surround sound, though the N64 was surround soundcapable.

\section{Player sounds}

As noted, the game features a wide diversity of player-usable objects, from weapons and gadgets to even a tank. Which many guns and gadgets share samples 
(again, Norgate only had 700k of available space for the audio), the game does provide a variety of useful semiotic distinctions that may serve to construct a sense of verisimilitude for the player, in spite of the game's lacking resolution. Importantly, the game lacks positional audio. However, projectile-other object collisions results in different sounds depending on the "material" of the other object. Glass, tile, and metal all have different sounds, and these sounds differ depending on the weapon used. Throwing knives for example, lightly ping and audibly bounce from material to material. Concerning the actual functions of the weapon, the gun clicks to notify the player that ammo is out and this same click sample is used when switching weapons. The game features no movement sounds and bodily sounds are limited to audible gasping when hit by an NPC. There is no real-time DSP used for player sounds.

\section{Non-player sounds}

NPC sounds offer some greater complexity than player sounds, including limited real-time DSP. Like player sounds, the game features a wide variety of different vehicles and weapons, some sharing the same samples. Other semiotic distinctions are similarly lacking. Like player sounds, movement sounds are nonexistent and bodily sounds are limited to grunts when hit. However, most notable for NPC sound is the use of proximity-dependent amplitude for NPC vehicles, weapons, and bodily sounds. For all of these, amplitude of the sample depends on how close the player is to the sound source. This is non-existent in multiplayer however, where all sounds are heard at the same volume, regardless of proximity — given the lack of online play or LAN possibilities, this is to be expected however, as players share the same screen. 


\section{Other sounds}

The game features no speech or dialog. Environmental sounds are limited to the sounds of doors opening and closing, though the amplitude of doors is dependent on player proximity and doors made of different materials sound different. There is no other environmental ambience present. Interface sounds, in terms of Grimshaw and Schott's (2007) taxonomy of diegetic positions, are positioned non-diegetically. The in-game interface is accessed via the player's wristwatch — an artifact of the Bond universe - and includes lo-fi static and computer-like clicking sounds. Continuing the FPS archetype set forth by Wolf $3 D$ (though such sounds were certainly not invented by the game's creators at id Software), GE 007 utilizes a variety of sounds to alert the player to "pick ups," including a reloading click when picking up ammo and a "zip" when picking up body armor. In terms of diegetic positioning, these sounds are also positioned non-diegetically. Finally, the music of the game is a looping score, similar to Wolf $3 D$, and is largely non-dynamic - i.e., it does not change dependent on game action, except upon the player's death.

\section{$\underline{\text { Summary }}$}

Overall, while the audio is a long way from contemporary games like $B F 3, G E$ 007 represents a significant departure from the ultra-simplistic sound design of Wolf $3 D$. In addition to various other innovations, perhaps most striking in the game's audio is its use of limited real-time DSP to adjust the amplitude of NPC sounds, depending on their proximity to the player, potentially inculcating both a sense of sensory immersion, this simulation of real-world acoustics can provide a the player a sense of presence in the world, but also challenge-based immersion in its anticipatory nature. 
Not only does the player trigger audio, such as gunfire, but the game's audio reacts to the player's movement throughout the map and is modified in real time, an important feature not found in earlier FPS games. Yet, still without positional audio, sounds all share the same diegetic position. While possibilities for identification with the avatar are augmented, the game's audio is still limited in many ways. The game's audio does provide more actionable information the player than Wolf $3 D$, but it is far removed from that to be found in the next sampled game, Halo: $C E$.

\section{Halo: Combat Evolved}

Halo: $C E$ was released in 2001 for the Xbox console by Bungie Studios. It has been heralded as one of the best FPS games, both visually and aurally. Sound design in the game is not merely a step up from GE 007; it is leagues beyond, in terms of diversity and mechanic complexity. Given a much more capable system, greater resources on the DVD-ROM disc versus the cartridge ROM of the N64, a larger audio team, and greater financial resources, this is not surprising. The game is surround sound-capable and includes both cooperative and versus multiplayer, as well as LAN possibilities for up to 16-player matches. As the game was released prior to Microsoft's online gaming service, Xbox Live, there is no official online multiplayer format. Various workarounds exist however to circumvent this limitation. Given that online multiplayer is not available in the game as-is, it has not been utilized in this study.

\section{Player sounds}

The game features fifteen weapons and five vehicles usable by the player, as well as three other vehicles not usable by the player. For the most part, these various 
objects are sonically unique, albeit with some overlap for similar weapons and vehicles. Given the first-person perspective, player sounds do not require positional audio. Vehicle sounds however do use positional audio as perspective switches to third-person when they are in use. Player vehicle sounds also offer a variety of semiotic distinctions, from the simple changing engine sound with acceleration, to differing tire-surface contact sounds, dependent on the material being driven on, as well as incidental "kicking up dirt" sounds, again, dependent on the material being driven on. In addition, different parts of vehicles produce sound, from suspension, to gun turrets, to doors opening and closing, providing a cohesive soundscape associated with each vehicle. Furthermore, these sounds vary as the vehicle takes on damage or makes contact with various surfaces in the virtual world. Notably however, vehicle sounds do not utilize real-time DSP dependent on location; i.e., driving in an enclosed space sounds no different than driving in an open canyon.

Like vehicle sounds, player weapon sounds are quite diverse and complex, featuring sounds beyond just the firing of the weapon. Most guns, both when fired or used in melee attacks, feature various reload sounds and other associated clicks and whirrs. The variety of sounds associated with a weapon are constructed as permutations of various foley sounds, ambient sounds, and audio samples, all dependent on the rate and location of fire. The result is rich and dynamic weapon sound that never truly repeats itself. Furthermore, utilizing real-time DSP, how the game deals with falling shell casings is quite complex and geared towards realistic representation, as lead audio designer Martin O'Donnell notes in an interview:

[...] the game keeps track of every shell casing and its impact velocity as well as the surface that it's impacting. We create a sound for the 
casing bounce, sweeten it with the sound of the impacted surface, and changes its amplitude with the velocity. That really helps to bring a level of reality to every physical thing that happens in Halo. (Marks \& O’Donnell, 2002)

This "level of reality" is brought about through other weapon sounds as well: utilizing real-time DSP, heavy machine guns, such as those equipped on the Warthog vehicle, among others, muffle other sounds due to how loud they are and nearby exploding grenades behave similarly, inducing even a virtual ear-ringing tinnitus in the player. Furthermore, the game audio makes various semiotic distinctions, not only between different weapons, but impacts sound different dependent on the material being impacted, including enemy bodies versus enemy shields and plasma rifles and handguns can overheat and produce certain sounds indicating they cannot be fired (as well as an audible gasp by the player). Movement sounds in the game are limited to the sounds of feet on the ground, though these sounds do change dependent on the material being walked upon. Finally, the game features only a small set of bodily sounds, including a melee arm "swoosh" sound and a diverse set of screams/grunts when dying.

\section{Non-player sounds}

NPC sounds introduce some additional levels of complexity, while still utilizing the aspects of real-time DSP from player sounds and the immense diversity of vehicle/weapon sounds and their associated sub-sounds. All NPC sounds are positioned in stereo space, depending on their diegetic position, or across the Dolby 5.1 surround sound system, if utilized. It should be noted however that surround sound in the game is not true 3D surround sound, which uses separate audio files for each 
channel. Halo: $C E$ makes use of the existent stereo files in outputting to a 5.1 system, as O’Donnell notes:

I figured that in the same way stereo sound fills up your car's four speakers, we could do the same with our stereo files. We simply took the stereo signal and sent $50 \%$ of it to the rears, and also to the LFO. The listener gets the sense of being in the middle of the ambient space and music, and therefore the sound of marines yelling or an explosion happening behind you is not jarring in any way. (O'Donnell, 2002)

The result is not a true surround sound experience; true 360-degree audio is barely approximated, but the result offers up a totally new dimension and sensory immersive possibility not found in GE 007 or Wolf $3 D$. In terms of real-time DSP, outside of shell dropping sounds, for vehicles, weapons, NPC movement, and NPC bodily sounds, amplitude varies based on proximity to the player. In terms of semiotic distinctions, movement sounds and bodily sounds, of which there exist many, vary based on the NPC type, allowing the player to identify the type of enemy using only aural cues.

\section{Other sounds}

The game features an immense amount of pre-recorded dialog, 6,200 lines to be exact (MacDonald, 2004). Some are a designed part of the campaign while many others are randomized and occur as part of the surrounding ambience. They are situated in either the stereo space or surround sound dependent on their origin location and their amplitude is dependent on their proximity to the player. Alert sounds are many and including a "reload" sound when picking up ammo, as well as various sounds foe camouflage pickups, overshield pickups, health pickups, and low/refilling life totals. In-game interface sounds are lacking; there are no click sounds and the 
music pauses. Perhaps most important of non-player and non-NPC sounds are environment sounds and the game's use of music.

Overall, the game provides a constant ambient atmosphere. Environmental sounds are many; just about any moving object in the game produces sound and seemingly never the same as any other sound. There are a wide variety of object collision sounds, door/portal sounds, and natural sounds, from rushing water, to chirping birds, to creaking trees, all situated in stereo or surround sound space and all with proximity-dependent amplitude. Conflated with the game's complex and dynamic music engine, these environmental sounds provide a constant, randomized ambience, where it is not just the samples themselves that are randomly permutated, but their tonal and dynamic ranges as well as where they are positioned in the soundscape; there are seemingly no instances of silence in the game. O’Donnell describes the music engine's dynamism:

It consists of three basic file types within a single soundtag. The "in", which starts the piece, the "loop" which is the middle section and plays for an indeterminate amount time, and the "out", which is how the piece ends. In addition, there is the "alt_loop", which plays instead of the loop if called by the game, and the "alt_out", which plays if the end is called during the alt_loop. The looping sections are made up of as many looping soundfiles as desired, and each looping soundfile can be of any length and weighted to determine the likelihood of being played. The level designer only needs to insert the commands: start, start_alt, and stop, in order to call any given music soundtag.

(O’Donnell, 2002)

In addition, similar soundtags were used on various object and other ambient background sounds, allowing the possibility to easily construct on-the-fly, dynamic soundscapes, bringing music and ambient sound together in a cohesive manner.

\section{$\underline{\text { Summary }}$}


Four years after the release of GE 007, Halo: $C E$ greatly expanded upon previous attempts at sonically immersive FPS experiences. With a much more capable system, expanded system resources, and a bigger budget, the game shed new light on what was possible in a console FPS in terms of audio design. Two particular aspects of the game merit further attention in the ways in which they (re)position the player in novel ways. First, the game's use of complex real-time DSP, as described at length above, not only replaces a traditionally repetitive soundscape with a dynamic and variable one, but it further emphasizes the mutual interaction between the player and the virtual world in which they inhabit. Second, and somewhat related to the first, the game's use of an underlying ambient soundscape, permutated via real-time DSP, implicates the entirety of the world's sounds, as well as the player's interactions with those sounds, in a much larger, cohesive soundscape. This feature was quite absent from the previously studied games, where the game's audio existed largely as isolated events. To re-quote Pete Parsons of Bungie, the studio behind Halo: $C E$, the new capabilities of the Microsoft Xbox, conflated with the novel audio design of the game, truly do change the way the genre is played: "In the past, people just stood there and blazed away until they were dead or they ran away. Now it's more of a game of I'm going to crouch down, find cover and stand up shoot [...] So ultimately this unnatural fusion of the controller and the screen they all continue to melt away" (as cited in Valdes, 2004). The result is a game that promotes immersive gameplay at all three of Ermi and Mäyrä's (2005) levels of immersion: it promotes sensory immersion via its use of real-time DSP and positional audio to inculcate a sense of presence in a world; it promotes challenge-based immersion also through these factors, as when conflated 
with NPC sounds, they become anticipatory and create a sense of tension in the player, which may result in attempts at stealth tactics; and it promotes imaginative immersion via the use of dynamic Player body sounds, but moreover via the game's ambient atmosphere and music, which sets the mood for the level segment.

\section{$\underline{\text { Battlefield } 3}$}

$B F 3$ was released in the Fall of 2011 by EA Digital Illusions CE (EA DICE), a full ten years after Halo: $C E$. Using the much-heralded and publicized Frostbite 2 game engine, the game's graphics, sound, and mechanics were met with critical acclaim. The game is fully surround sound capable with Dolby 5.1 and allows for a single-player campaign mode, as well as a wide variety of online multiplayer modes, including up to 12-on-12 games, with full voice-over-IP (VOIP) functionality. This study details only the original $B F 3$, without the various downloadable expansion packs. BF3 features 58 different weapons, with a multitude of available attachments, 23 various gadgets, 22 usable vehicles, 4 sprawling multiplayer maps, and 12 massive campaign missions, all with their own unique sonic profiles. The shear amount of audio assets in the game - a number that has not been publicized, in an extensive search of marketing, critical reviews, and developer interviews — presents an immense descriptive challenge

\section{Player sounds}

Unlike on-foot navigation, vehicles allow for both first- and third-person perspective, and given that they allow for perspective shifting and rotation, they also allow for positional audio. As noted, the game allows for true Dolby 5.1 surround sound, but even in a stereo setup, surround sound is simulated, placing sound 
vertically and horizontally in stereo space, and at varying amplitudes, to simulate $360-$ degree sound. Through modeling real-world head-related transfer functions (HRTFs), i.e., how the ear spatializes sound, even a stereo setup allows for the player to localize sound sources behind them, such as the roar of their F/A 18 Super Hornet's engine. In addition to utilizing positional audio, vehicles features an immense variety of semiotic distinctions, such as beeping in any of the fighter jets or tanks when an enemy missile is locked on, or beeping when armor is reaching critically low levels.

Weapon sounds, like all sounds in the game, feature complex real-time DSP that introduces acoustic effects synchronous with the implied environment; a single round shot from the semi-automatic MK 11 rifle will sound entirely different when fired inside of a concrete building rather than an open field. With the introduction of real-time dynamic reverb and amplitude modulation, weapon sounds, as well as most other sounds in the game, take on the acoustic profile of the environment of which they originate. Even subtle differences can be perceived, such as the slight reverberation increase of a weapon fired in a concrete room over a weapon fired in a wooden shack. This is certainly not the only role taken by the game engine's real-time DSP, but, unlike Halo: $C E$, its functions and underlying mechanics have not been detailed at length in press. Stefan Strandberg, the game's audio director, does point to the following goal of real-time DSP throughout the game's predecessor, Battlefield: Bad Company 2 (Electronic Arts, 2010): "It is so easy to break immersion, so we work hard to counteract repetition, because a game by nature is repetition. So a lot of sounds do have factors of dynamically generated manipulation applied to them" (Destructoid, 2010). It is unlikely that goal was done away with in the series' recent incarnation, and 
if anything it has become more possible, especially at the sensory level. Gunfire is wholly dynamic. While samples are certainly reused, they are modulated in various ways and randomized in various permutations so that they very rarely, if ever, repeat themselves. Coupled with this dynamic manipulation are the variety of "incidental" weapon sounds, such as reload clicking, falling shells, strap movement, sight adjustment, and the variety of other weapon sounds that don't involve actually firing the weapon. Furthermore, a variety of semiotic distinctions complete the sonic profile of these weapons, including manipulation based on the material of the object hit, “pinging” when weapons overheat or are close to overheating, explosion-induced simulated tinnitus, and the muffling of other sounds when machine guns and light machine guns are fired nearby.

Movement sounds and bodily sounds in the game take on a similar level of richness and complexity, utilizing the same real-time DSP, and helping to promote a sense of imaginative immersion at level not found in Halo: CE. Unlike any of the previous game's studied thus far, $B F 3$ features a wide-variety of movement sounds, from the rustling of clothing when moving, crouching, and aiming down sights, to dynamic feet-ground movement sounds, variable based on speed and surface material, to the different sounds of melee attacks, dependent on the weapon used and the object hit. Like weapon sounds, these sounds are fully randomized and expressed in permutations as to never seem repetitive. Bodily sounds include variable breathing rates, especially when aiming through a sniper scope — the player must hold their breath in order to take a steady shot — as well as a diverse array of grunts and groans 
when moving and when injured or killed. Overall, it is difficult to exaggerate the complexity and richness of the game's Player sounds.

\section{$\underline{\text { Non-player sounds }}$}

NPC sounds utilize the same diversity and real-time DSP for vehicle sounds, weapon sounds, movement sounds, and bodily sounds. Rather than just vehicle sounds however, all NPC sounds are localized in 3D space, either in true Dolby 5.1 surround sound or simulated surround sound in stereo format. This factor is of supreme importance for gameplay. Based on the conflation of 3D sound and real-time DSP, as well as the immense diversity of sounds, a player can be signaled to imminent danger emanating from all around them. Nearby movement, gunfire, vehicle sounds, and dialog can all be localized as originating from a particular direction, and, based on amplitude, the player can even estimate their proximity. While this was somewhat possible in Halo: $C E$, it was to nowhere near the degree as it is in BF3. The importance of this to the possibilities of an immersive gameplay experience cannot be exaggerated.

\section{Other sounds}

Nearly triple the amount from Halo: $C E, B F 3$ features around 18,000 audio assets pertaining to speech and dialog (Veselka, 2011). As in Halo: CE, dialog is used to propel the narrative in the first-person campaign and provide for the overall sonic ambience of the game - entire conversations, wholly unrelated to the campaign, will take place between AI-controlled NPCs, if the player chooses to stick around and listen. However, in addition, dialog takes on a much more supportive, functional role in $B F 3$. In multiplayer formats, user-generated dialog provides cues to the player and 
human-controlled NPCs via the game's "spotting" mechanic and the ability to ask for different types of assistance and give commands to your squad - a prime example of Grimshaw and Schott's (2007) telediegetic positioning, where sounds produced by other players affect the current player. In multiplayer modes, players take on a particular combat class - Assault, Engineer, Recon, and Support — and each of these classes has a particular role to play in the game, from supplying medical assistance in the assault class, to designating targets in the recon class. These roles can give audio commands to squadmates and indicate when an enemy is seen — "spotting." This voiceover dialog is sent over the radio and takes on dynamic static in the simulation of a radio exchange. In addition, in the single-player campaign mode, as the NPC who is speaking over the radio is neared, their unaltered voice is mixed in with the radio transmission. The same types of real-time DSP and positional audio present in player and NPC sounds are also utilized. Triggers however don't work perfectly; in the single-player campaign mode, if the game expects the player to be in a location, it will trigger friendly NPC in-person voiceovers even if the player is not present. The majority of game dialog occupies an alert-type function, but various non-vocal alert sounds also exist, such as clicking and other collision-type sounds when picking up ammo or medical kits, occupying an enemy territory, or when a squadmate spawns nearby.

Like Halo: $C E, B F 3$ provides an overall ambient soundscape, densely packed with distant gunfire, natural sounds, bullet ricochet, distant chatter, and explosions, all of which is located in 3D space and subject to a variety of real-time DSP manipulation, all assisting in inculcating a sense of imaginative immersion in the 
player. Like weapon and other object-interaction sounds, these sounds are randomized and presented in varying permutations so as to never seem repetitive. In addition, certain soundscapes are linked to certain spaces — the ambient outdoor sounds of a wide-open valley become lower in the mix when a building is entered and a new set of ambient sounds arise, though the former never seem to diminish fully. Similarly, levels in both single-player and multiplayer game modes are characterized by particular ambiences, as Strandberg notes (again, specific to Battlefield: Bad Company 2, though the series' most recent incarnation hardly differs in this regard):

The ambiances we have in Bad Company 2 do much more than just provide background noise for the other noises to sit in [...] [We put] a type of narrative in the ambiance for each level. They are made to provoke a feeling that resonates with the current stage in the singleplayer narrative. It works probably mostly subconsciously; by putting extra effort and thought into the design, there is a lot to gain. (as cited in Destructoid, 2010)

The game's music, or lack thereof, contributes to its ambience. There is very little in-game music, unlike Halo: $C E$. The music that does exist tends to take on a triggered "alert" role rather than an atmospheric role, such as in multiplayer game modes as the match nears its end or in the single-player campaign when a mission is failed. Music is used in the game's pre- and post-mission menus, but it is sparse and atmospheric. This interface features a simple click when selecting options and is in no way metaphorical like the interface in GE 007. Somewhat different however is the ingame multiplayer interface; while choosing a class at the beginning of a match or upon death, real-time match sounds are heard, but they are muffled. Thus while the design of the interface may not be metaphorical and non-metaphorical "clicks" are still 
present, there is still a connection to the soundscape of the present match; the player is never truly severed from the game.

\section{$\underline{\text { Summary }}$}

A full twenty years removed from Wolf $3 D, B F 3$ heralds sound design that, better than ever before, emulates first-person audition — or at least audition as it has been constructed in war cinema. With full 360-degree positional audio, either in Dolby 5.1 or simulated surround sound, and complex real-time DSP, the game can offer the player an experience analogous to real-world audition, promoting an immersive aural experience to coincide with the visual. As with Halo: $C E$, where the game truly finds itself removed from earlier attempts is in its cohesive soundscape. If the player takes a minute to just listen in a 12-on-12 multiplayer match, perhaps at the edge of the map where NPCs concerned with victory seldom lurk, they will be presented with an incredibly rich, diverse soundscape, seldom, if ever, verging on repetitive. As Standberg has asserted, the game's sound design "is not about creating an awesome gun sound; it's about creating a war" (as cited in Tong, 2011). To use the language of Truax (2001), this ambience provides a sonic "ground" on which the various "figure" sounds of gunfire and NPC chatter can find support. With this ground, the figure sounds feel out of place, disjointed, in relation to lived aural experience where noise hardly, if ever, ceases. At all levels of Ermi and Mäyrä’s (2005) levels of immersion, the game serves to promote an immersive experience. While sensory immersion and imaginative immersion are brought to a level not found in Halo: $C E$ or the other previous games studied, this is perhaps most evident in challenge-based immersion. Multiplayer gameplay modes inculcate this especially so; as the player moves about 
the map, putting the game's complex real-time DSP and positional audio to use, the game's mechanics and map design facilitate avoidance and stealth tactics. The Recon class provides an excellent example of this, geared towards a sniping tactic of gameplay where the player hides or moves around the map as silently as possible. In such a mode, sound plays a major role, alerting to the player to other players sneaking up behind them if they are sniping, or nearby NPCs if they are moving around the map stealthily. While the aesthetic strategy of the game has been to promote an audiovisually realistic experience and inculcate immersion through this aesthetic of realism, the game's audio is also eminently functional. Sounds, from weapon sounds to the game's ambient background noise to ammo pickup alerts, are not merely present for their own sake; they are interactive and actionable. While a viewer of war cinema may find themselves inhabiting a fictional world, $B F 3$ positions the player as such that they not merely inhabit a world, but that they are an interactant within it. 


\section{BIBLIOGRAPHY}

1UP. (n.d.). The 60 most influential games of all time. 1UP. Retrieved January 15, 2014, from http://www.1up.com/features/most-influential-games?pager.offset=2

Aarseth, E. (1997). Cybertext: Perspectives on ergodic literature. Baltimore, MD: Johns Hopkins University Press.

Aarseth, E. (2001). Computer game studies, year one. Game Studies, 1(1). Retrieved January 15, 2014, from http://www.gamestudies.org/0101/editorial.html

America's Army: Operations. (2001). United States Army.

Apogee Software. (1995). Attention Wolfenstein 3D fans! [Press release]. Retrieved January 15, 2014, from www.3Drealms.com/wolf3D/w3D_su_advert.pdf

Applied Acoustics Systems. (n.d.). Sample rate \& bit depth. Retrieved March 01, 2014, from https://www.applied-acoustics.com/techtalk/sampleratebitdepth/

Asteroids. (1979). Sunnyvale, CA: Atari, Inc.

Atkins, B. (2003). More than a game: The computer game as fictional form. Manchester, UK: Manchester University Press.

Attali, J. (1985). Noise: The political economy of music. Minneapolis, MN: University of Minnesota Press.

Avedon, E. M., \& Sutton-Smith, B. (1971). The study of games. New York, NY: Wiley-Blackwell.

Baron, J. (2010). Digital historicism: archival footage, digital interface, and historiographic effects in Call of Duty: World at War. Eludamos. Journal for Computer Game Culture, 4(2), 303-314. Retrieved January 15, 2014, from http://www.eludamos.org/index.php/eludamos/article/viewArticle/96

Barr, P. (2008). Video game values: Play as human-computer interaction [Doctoral dissertation]. Wellington. Retrieved January 15, 2014, from http://www.pippinbarr.com/academic/Pippin_Barr_PhD_Thesis.pdf

Battlefield 3. (2011). Stockholm, Sweden: EA Digital Illusions CE.

Battlefield: Bad Company 2. (2010). Redwood City, CA: Electronic Arts. 
Battlezone. (1980). Sunnyvale, CA: Atari, Inc.

Baudrillard, J. (1995). The Gulf War did not take place. Bloomington, IN: Indiana University.

Bogost, I. (2008). The rhetoric of video games. In K. Salen (Ed.), The ecology of games: Connecting youth, games, and learning (pp. 117-139). Cambridge, MA: MIT Press. doi:10.1162/dmal.9780262693646.117

Bogost, I. (2011). How to do things with videogames. Minneapolis, MN: University of Minnesota Press.

Boulding, A. (2001). Halo review. IGN. Retrieved January 15, 2014, from http://www.ign.com/articles/2001/11/10/halo-review?page=1

Breakout. (1976). Sunnyvale, CA: Atari, Inc.

Brown, M. (2013). Life as a US drone operator: "It"s like playing a video game for four years'. The Guardian. Retrieved January 15, 2014, from http://www.theguardian.com/world/2013/jul/28/life-us-drone-operator-artist

Bull, M. (2000). Sounding out the city: Personal stereos and the management of everyday life. New York, NY: Oxford University Press.

Cage, J. (1967). A Year From monday: New lectures and writings. Middletown, CT: Wesleyan University Press.

Call of Duty: World at War. (2008). Santa Monica, CA: Activision Blizzard.

Callois, R. (2001). Man, play and games. Champaign, IL: University of Illinois Press.

Catacomb 3-D. (1991). Richardson, TX: id Software.

Cheung, S. (2012). The un-realism of first person shooters. Gizmo insider. Retrieved January 15, 2014, from http://gizmoinsider.com/the-un-realism-of-first-personshooters-editorial-922815.html

Chion, M. (1994). Audio-vision: Sound on screen. New York, NY: Columbia University Press.

Chion, M. (2009). Film, a sound art. New York, NY: Columbia University Press.

Christensen, N. C. (2006). Geeks at play: Doing masculinity in an online gaming site. Reconstruction, 1(1). Retrieved January 15, 2014, from http://reconstruction.eserver.org/061/christensen.shtml 
CODiE. (n.d.). Past CODiE award winners. Retrieved January 15, 2014, from http://www.siia.net/codies/2010/pw_1993.asp

Collins, K. (2008). Game sound: An introduction to the history, theory, and practice of video game music and sound design (Kindle ver.). Cambridge, MA: MIT Press.

Corbin, A. (1998). Village bells: Sound and meanng in the nineteenth-century French countryside. New York, NY: Columbia University Press.

D'Angelo, W. (2012). Top 10 in sales - First person shooters. VGChartz. Retrieved January 15, 2014, from http://www.vgchartz.com/article/250080/top-10-in-salesfirst-person-shooters/

Dactyl Nightmare. (1991). Virtuality.

Davison, B., \& Walker, B. N. (2008). AudioPlusWidgets: Bringing sound to software widgets and interface components. In Proceedings of the International Conference on Auditory Display (ICAD 2008). Paris.

Defender. (1980). New York, NY: Williams Electronics.

Delta Six. (n.d.). Delta Six. Retrieved January 15, 2014, from http://www.thedeltasix.com/

Designing Sound. (2010). From N64 to Wii, re-imagining "GoldenEye 007." Designing Sound. Retrieved January 15, 2014, from http://designingsound.org/2010/11/from-n64-to-wii-re-imagining-goldeneye-007exclusive-interview-with-graeme-norgate-and-steve-duckworth/

Destructoid. (2010). A powerful ally for narrative: The audio of Bad Company 2. Destructoid. Retrieved January 15, 2014, from http://www.destructoid.com/apowerful-ally-for-narrative-the-audio-of-bad-company-2-164725.phtml

Dillon, R. (2011). The golden age of video games: The birth of a multibillion dollar industry. Boca Raton, FL: A.K. Peters/CRC Press.

Donovan, T. (2010). Replay: The history of video games. East Sussex, UK: Yellow Ant.

Doom. (1993). Richardson, TX: id Software.

Droumeva, M. (2011). An acoustic communication framework for game sound: Fidelity, versimilitude, ecology. In M. Grimshaw (Ed.), Game sound technology and player interaction: Concepts and developments (pp. 131-152). Hershey, PA: IGI Global. 
Dyck, J., Pinelle, D., Brown, B., \& Gutwin, C. (2003). Learning from games: HCI design innovations in entertainment software. In Proceedings of the 2003 Conference on Graphics Interface.

EA DICE. (2011a). TV Launch Trailer [Television commercial]. Retrieved January 15, 2014, from http://www.battlefield.com/battlefield3/videos/is-it-real-trailer

EA DICE. (2011b). My Life [Television commercial]. Retrieved January 15, 2014, from http://www.youtube.com/watch?v=TfrrAp1blaM

Ermi, L., \& Mäyrä, F. (2005). Fundamental components of the gameplay experience: Analysing immersion. In DIGRA Conf.

Eskelinen, M. (2004). Towards computer game studies. Electronic book review. January 15, 2014, Retrieved from http://www.electronicbookreview.com/thread/firstperson/anticolonial

Eykemans, P. (2011). Battlefield 3 review. IGN. Retrieved January 15, 2014, from http://www.ign.com/articles/2011/10/26/battlefield-3-review-2

Fabricatore, C., Nussbaum, M., \& Rosas, R. (2002). Playability in action videogames: A qualitative design model. Human-Computer Interaction, 17(4), 311-368.

Ferguson, C. J., \& Olson, C. K. (2014). Video game violence use among "vulnerable" populations: The impact of violent games on delinquency and bullying among children with clinically elevated depression or attention deficit symptoms. Journal of Youth and Adolescence, 43(1), 127-136. doi:10.1007/s10964-0139986-5

Fernstrom, M. (2005). HCI design and interactive sonification for fingers and ears. MultiMedia, IEEE, 12(2), 36-44.

Fielder, J. (2001). Halo Review. Gamespot. Retrieved January 15, 2014, from http://www.gamespot.com/reviews/halo-review/1900-2823816/

Filiciak, M. (2003). Hyperidentities: Postmodern identity patterns in massively multiplayer online role-playing games. In M. J. P. Wolf \& B. Perron (Eds.), The video game theory reader. New York, NY: Routledge.

Folmann, T. (2004). Dimensions of game audio. Retrieved January 15, 2014, from http://troelsfolmann.blogspot.com/2004/11/dimensions-of-game-audio.html

Frasca, G. (2003). Ludologists love stories , too: Notes from a debate that never took place. Ludology. Retrieved January 15, 2014, from http://ludology.org/articles/Frasca_LevelUp2003.pdf 
Friberg, J., \& Gärdenfors, D. (2004). Audio Games: New perspectives on game audio. In Proceedings of the 2004 ACM SIGCHI International Conference on Advances in computer entertainment technology. Association for Computing Machinery. Retrieved from Proceedings of the 2004 ACM SIGCHI International Conference on Advances in computer entertainment technology

Fritsch, M. (2013). History of video game music. In P. Moormann (Ed.), Music and games: Perspectives on a popular alliance (pp. 11-41). Wiesbaden: Springer VS.

Fulton, B. (2002). Beyond psychological theory: Getting data that improves games. Gamastura. Retrieved from January 15, 2014, http://www.gamasutra.com/view/feature/3003/beyond_psychological_theory_ph $\mathrm{p}$

G4. (2012). Best of 2011: Best sound design. Retrieved January 15, 2014, from http://www.g4tv.com/videos/56652/best-of-2011-best-sound-design/

Game Developers Conference. (2013). GDC Awards to honor Spacewar!'s Steve Russell, Smithsonian curator Chris Melissins. GDC news and information blog. Retrieved January 15, 2014, from http://www.gdconf.com/news/gdc_awards_to_honor_spacewars_html

Game Informer. (2011). The sound design of Battlefield 3. Retrieved January 15, 2014, from http://www.youtube.com/watch?v=Vc8WQsIxhro

Gamespot. (2012). The best of 2011 special achievments: Best sound design. Gamespot. Retrieved January 15, 2014, from http://www.gamespot.com/best-of2011-special/awards/index.html?page $=11$

Gerstmann, J. (1997). GoldenEye 007 review. Gamespot. Retrieved January 15, 2014, from http://www.gamespot.com/reviews/goldeneye-007-review/1900-2544509/

Gieselmann, H. (2007). Ordinary gamers - The vanishing violence in war games and its influence on male gamers. Eludamos. Journal for Computer Game Culture, I(1). Retrieved January 15, 2014, from http://www.eludamos.org/index.php/eludamos/article/viewArticle/3

GO-Gulf. (2012). Smartphone users around the world - statistics and facts [Infographic]. GO-Gulf. Retrieved January 15, 2014, from http://www.gogulf.com/blog/smartphone/

GoldenEye 007. (1997). Leicestershire, England: Rare.

Grimshaw, M. (2007a). Sound and immersion in the first-person shooter. Retrieved January 15, 2014, from 
http://wlv.openrepository.com/wlv/bitstream/2436/35995/2/Grimshaw_CGAME S07.pdf

Grimshaw, M. (2007b). Acoustic ecology of the first-person shooter [Doctoral dissertation]. Waikato, NZ. Retrieved January 15, 2014, from http://www.wikindx.com/mainsite/acousticEcologyOfTheFirstPersonShooter.pdf

Grimshaw, M., Charlton, J. P., \& Jagger, R. (2011). First-person shooters : Immersion and attention. Eludamos. Journal for Computer Game Culture, 5(1). Retrieved January 15, 2014, from http://www.eludamos.org/index.php/eludamos/article/viewArticle/vol5no13/html3

Grimshaw, M., \& Schott, G. (2007). Situating gaming as a sonic experience : The acoustic ecology of first-person shooters. In Situated Play, Proceedings of DiGRA 2007 Conference (pp. 474-481). Retrieved January 15, 2014, from http://www.digra.org/digital-library/publications/situating-gaming-as-a-sonicexperience-the-acoustic-ecology-of-first-person-shooters/

Halo: Combat Evolved. (2001). Bellevue, Washington: Bungie.

Huiberts, S. (2010). Captivating sound: The role of audio for immersion in computer games [Doctoral dissertation]. Utrecht, The Netherlands. Retrieved January 15, 2014, from

http://download.captivatingsound.com/Sander_Huiberts_CaptivatingSound.pdf

Huizinga, J. (1971). Homo ludens: A study of the play-element in culture. Boston, MA: Beacon Press.

Hyperkin. (n.d.). “ComRad” wireless gaming audio headset. Retrieved January 15, 2014, from http://hyperkin.com/ps3-xbox-360-pc-comrad-wireless-gamingaudio-helmet-hyperkin.html

Ibrahim, A. A., \& Hunt, A. (2007). An HCI model for usability of sonification applications. In K. Coninx, K. Luyten, \& K. A. Schneider (Eds.), Task models and diagrams for users interface design. New York, NY: Springer.

IGN. (n.d.). ign's top 100 games of all time. Retrieved January 15, 2014, from http://uk.top100.ign.com/2007/ign_top_game_62.html

Ihde, D. (2007). Listening and voice. Albany, NY: SUNY Press.

Jorgensen, F. (2012). The top 10 most immersive games ever made. Daxgamer. Retrieved January 15, 2014, from http://daxgamer.com/2012/03/top-10immersive-games/3/ 
Just Claws. (n.d.). Wolfenstein 3D [Press release]. Retrieved January 15, 2014, from http://justclaws.atari.org/jagsite/gameinfo/wolf3D.htm

Juul, J. (n.d.). A history of the computer game. Retrieved January 15, 2014, from https://www.jesperjuul.net/thesis/2-historyofthecomputergame.html

Juul, J. (2005). Half-real: Video games between real rules and fictional worlds. Cambridge, MA: MIT Press.

Kaae, J. (2008). Theoretical approaches to composing dynamic music for video games. In K. Collins (Ed.), From Pac-Man to pop music: Interactive audio in games and new media (pp. 75-92). Surrey: Ashgate.

Kent, S. L. (2001). The ultimate history of video games: From Pong to Pokemon. Roseville, CA: Prima.

Kotcheff, T. (1982). First blood. United States of America: Orion Pictures.

Kücklich, J. (2003). Perspectives of computer game philogy. Game Studies, 3(1). Retrieved January 15, 2014, from http://www.gamestudies.org/0301/kucklich/

Langkjaer, B. (2009). Making fictions sound real - On film sound, perceptual realism and genre. MedieKultur: Journal of Media and Communication Research, 26(48). Retrieved January 15, 2014, from http://ojs.statsbiblioteket.dk/index.php/mediekultur/article/view/2115

Latour, B. (1993). We have never been modern. Cambridge, MA: Harvard University Press.

Lazzaro, N., \& Keeker, K. (2004). What's my method?: A game show on games. In CHI'04: Extended Abstracts of the 2004 Conference on Human Factors and Computing Systems (pp. 1093-1094). ACM Press.

Lowood, H. (2006). Game studies now, history of science then. Games and Culture, 1(1), 78-82. doi:10.1177/1555412005281404

MacDonald, M. (2004). Marty O’Donnell interview. 1UP. Retrieved January 15, 2014, from http://www.1up.com/previews/marty-oonnell-interview

Mackey, B. (n.d.). The Essential 100, No. 23: Wolfenstein 3D. 1UP. Retrieved January 15, 2014, from http://www.1up.com/features/essential-23-wolfenstein-3D

Malone, T. W. (1982). Heuristics for designing enjoyable user interfaces: Lessons from computer games. In CHI '82: Proceedings of the 1982 Conference on Human Factors in Computing Systems (pp. 63-68). ACM Press. 
Markham, X. (2012). 15 year anniversary retrospective: GoldenEye 007. Gamastura. Retrieved January 15, 2014, from http://www.gamasutra.com/blogs/XanderMarkham/20120825/176550/15_Year_ Anni versary_Retrospective_GoldenEye_007_N64_1997.php

Marks, A., \& O'Donnell, M. (2002). The use and effectiveness of audio in Halo: Combat Evolved. Music4games. Retrieved January 15, 2014, from http://web.archive.org/web/20060619120737/http://www.music4games.net/Featu res_Display.aspx?id=24

Mazel, J. (2009). The 50 best selling videogames of the 1990s worldwide. VGChartz. Retrieved January 15, 2014, from http://www.vgchartz.com/article/4145/the-50best-selling-videogames-of-the-1990s-worldwide/

McMahan, A. (2003). Immersion, engagement, and presence: A new method for analyzing 3-D games. In M. J. P. Wolf \& B. Perron (Eds.), The video game theory reader (pp. 66-87). New York, NY.

Medal of Honor: Warfighter. (2012). Redwood City, CA: Electronic Arts.

Melissinos, C., \& O'Rourke, P. (2012). The art of video games: From Pac-man to Mass Effect. New York, NY: Welcome Books.

Metacritic. (n.d.-a). GoldenEye 007. Metacritic. Retrieved January 15, 2014, from http://www.metacritic.com/game/nintendo-64

Metacritic. (n.d.-b). Halo: Combat Evolved. Metacritic. Retrieved January 15, 2014, from http://www.metacritic.com/game/xbox/halo-combat-evolved

Metacritic. (n.d.-c). Battlefield 3. Metacritic. Retrieved January 15, 2014, from http://www.metacritic.com/game/playstation-3/battlefield-3

Metz, C. (1974). Film language: A semiotics of cinema. New York, NY: Oxford University Press.

Microsoft. (n.d.). Experience the Xbox One. Xbox. Retrieved January 15, 2014, from http://www.xbox.com/en-US/xbox-one/meet-xbox-one\#adrenalinejunkie

Microsoft. (2002). "Halo: Combat Evolved" for Xbox tops 1 million mark in record time. Microsoft news center. Retrieved January 15, 2014, from http://www.microsoft.com/en-us/news/press/2002/apr02/04-08halomillionpr.aspx

Monfort, N., Baudoin, P., Bell, J., Bogost, I., Douglass, J., Marino, M. C., ... Vawter, N. (2013). 10 PRINT CHR $\$(205.5+R N D(1)) ;:$ GOTO 10. Cambridge, MA: MIT Press. 
Montfort, N., \& Bogost, I. (2009). Racing the beam: The Atari Video Computer System. Cambridge, MA: MIT Press.

Murray, J. H. (1997). Hamlet on the holodeck: The future of narrative in cyberspace. Cambridge, MA: MIT Press.

Nielsen, H. S. (2010). The computer game as a somatic experience. Eludamos. Journal for Computer Game Culture, 4(1), 25-40. Retrieved January 15, 2014, from http://www.eludamos.org/index.php/eludamos/article/viewArticle/80/147

Nintendo. (1995). Tomorrow Never Knows [Television commercial]. Retrieved January 15, 2014, from http://www.youtube.com/watch?v=FtHNtw1hXVY

Nintendo Power. (1994). Now playing. Nintendo Power, 58, 102-107.

O’Donnell, M. (2002). Producing audio for halo. Gamasutra. Retrieved January 15, 2014, from

http://www.gamasutra.com/view/feature/3489/producing_audio_for_halo.php

Oculus VR. (n.d.). Oculus Rift. Retrieved January 15, 2014, from http://www.oculusvr.com/

Pagulayan, R. J., Keeker, K., Wixon, D., Romero, R. L., \& Fuller, T. (2003). Usercentered design in games. In J. A. Jacko \& A. Sears (Eds.), The human-computer interaction handbook: Fundamentals, evolving technologies, and emerging applications (pp. 884-906). Mahwah, NJ: Lawrence Erlbaum Associates.

Perry, D. (1997). GoldenEye 007. IGN. Retrieved January 15, 2014, from http://www.ign.com/articles/1997/08/26/goldeneye-007

Pinch, T., \& Bijsterveld, K. (2004). Sound studies: New technologies and music. Social Studies of Science, 34(5), 635-648.

Poitras, L., Rosenbach, M., \& Stark, H. (2013). Ally and target: US intelligence watches Germany closely. Der Spiegel Online. Retrieved January 15, 2014, from http://www.spiegel.de/international/world/germany-is-a-both-a-partner-to-and-atarget-of-nsa-surveillance-a-916029.html

Pong. (1972). Sunnyvale, CA: Atari, Inc.

Rainbow Six Vegas 2. (2008). Montreuil, France: Ubisoft.

Reiner, M., \& Hecht, D. (2009). Behavioral indications of object-presence in haptic virtual environments. Cyberpsychology \& Behavior, 12(2), 183-186. 
Reyes, I., \& Adams, S. (2010). Screening play: Rules, wares, and representations in "realistic" video games. Eludamos. Journal for Computer Game Culture, 4(2), 149-166. Retrieved January 15, 2014, from http://www.eludamos.org/index.php/eludamos/article/viewArticle/vol4no2-3

Rowlands, T. E. (2010). Empire of the hyperreal: A critical ethnography of "EverQuest" [Doctoral dissertation]. Arizona State University. Retrieved January 15, 2014, from http://gradworks.umi.com/34/10/3410628.html

Saether, B. E. (2011). How to become a better player in Battlefield 3 multiplayer. RespawnLess. Retrieved January 15, 2014, from http://www.respawnless.com/battlefield-3-multiplayer-guide/become-a-betterplayer-in-battlefield-3-multiplayer/

SAY Media. (2011). Combat Evolved Anniversary 3D Effect Online Ad [Press release]. Retrieved January 15, 2014, from http://www.saymedia.com/news/combat-evolved-anniversary-3D-effect-onlinead-campaign

Schafer, R. M. (1977). The soundscape: Our sonic environment and the tuning of the world. Rochester, VT: Destiny Books.

Shimpi, A. L. (2013). The Haswell review: Intel Core i7-4770K \& i5-4670K tested. Anandtech. Retrieved January 15, 2014, from http://www.anandtech.com/show/7003/the-haswell-review-intel-core-i74770ki54560k-tested/5

SIA. (n.d.). 2000-1992 Shareware Industry Awards winners. Retrieved January 15, 2014, from http://web.archive.org/web/20100214083926/http:/sia.sic.org/pastwinners.php

Silverman, K. (1983). The Subject of Semiotics. New York, NY: Oxford University Press.

Smith, A. (2013). Smartphone Ownership 2013. Pew Internet. Retrieved January 15, 2014, from http://pewinternet.org/Reports/2013/Smartphone-Ownership2013/Findings.aspx

Smith, M. (2001). Listening to nineteenth-century America. Chapel Hill, NC: University of North Carolina Press.

Sofge, E. (2008). Shooting for realism: How accurate are video-game weapns? Popular Mechanics. Retrieved January 15, 2014, from http://www.popularmechanics.com/technology/gadgets/video-games/4255750 
Sony. (n.d.). PS4 Greatness Awaits. PS4. Retrieved January 15, 2014, from http://us.playstation.com/ps4/index.htm

Sterne, J. (2003). The audible past: Cultural origins of sound reproduction. Durham, NC: Duke University Press.

Stockburger, A. (2003). The game environment from an auditive perspective. Audo Games. Retrieved January 15, 2014, from http://audiogames.net/pics/upload/gameenvironment.htm

Tetris. (1989). Kyoto, Japan: Nintemdo.

The Game Effect. (2012). TGE GOTY awards 2011: Best sound design. The Game Effect. Retrieved January 15, 2014, from http://www.thegameeffect.com/awards/game-of-the-year-awards-2011/bestsound-design

Thelen, E. (2004). PDP-1. Retrieved January 15, 2014, from http://edthelen.org/comp-hist/pdp-1.html

Thomsen, M. (2012). The unsettling realism of 2012's two big first-person shooter games. The Atlantic. Retrieved January 15, 2014, from http://www.theatlantic.com/entertainment/archive/2012/12/the-unsettlingrealism-of-2012s-two-

Tol, B. R. Van, \& Huiberts, S. (2008). IEZA : A framework for game audio typologies for game audio. Gamastura. Retrieved January 15, 2014, from http://www.gamasutra.com/view/feature/3509/ieza_a_framework_for_game_audi o.php

Tong, S. (2011). Sound byte: Meet the audio director of Battlefield 3. Gamespot. Retrieved January 15, 2014, from http://www.gamespot.com/articles/sound-bytemeet-the-audio-director-of-battlefield-3/1100-6307723/

Truax, B. (2001). Acoustic communication. Westport, CT: Ablex Publishing.

Valdes, R. (2004). Halo 2 sound. Howstuffworks. Retrieved January 15, 2014, from http://electronics.howstuffworks.com/halo-sound.htm

Veselka, D. (2011). Massive map sizes in Battlefield 3 and more on sound design. MP1st. Retrieved January 15, 2014, from http://mp1st.com/2011/09/26/vo-linesin-battlefield-3-and-massive-map-sizes/

Veterans Benefits Administration. (2011). Annual benefits report: Fiscal year 2011. Retrieved January 15, 2014, from http://www.vba.va.gov/REPORTS/abr/2011_abr.pdf 
VGChartz. (n.d.-a). GoldenEye 007. VGChartz. Retrieved January 15, 2014, from http://www.vgchartz.com/game/877/goldeneye-007/

VGChartz. (n.d.-b). Halo: Combat Evolved. VGChartz. Retrieved January 15, 2014, from http://www.vgchartz.com/game/939/halo-combat-evolved/

VGChartz. (n.d.-c). Battlefield 3. VGChartz. Retrieved January 15, 2014, from http://www.vgchartz.com/game/40230/battlefield-3/

Walker, B. N. (2013). Sonification and auditory displays in electronic devices. In Proceedings of the Sound and Music Computing Conference (SMC2013), Stockholm. Stockholm.

Watters, C. (2011). Battlefield 3 Review. Gamespot. Retrieved January 15, 2014, from http://www.gamespot.com/reviews/battlefield-3-review/1900-6342626/

Webster, A. (2013). How Atari box art turned 8-bit games into virtual wonderlands. The Verge. Retrieved January 15, 2014, from http://www.theverge.com/2013/9/19/4716444/how-atari-box-art-turned-8-bitgames-into-virtual-wonderlands

Weske, J. (2002). Digital sound and music in video games. Retrieved January 15, 2014, from http://3Daudio.info/gamesound/index.html

Whalen, Z. (2001). Play along: An approach to video game music. Game Studies, 4(1). Retrieved January 15, 2014, from http://www.gamestudies.org/0401/whalen/

Whitehead, T. (2013). GoldenEye creator explains N64 title's role in bringing "real" guns to the virtual world. Nintendo Life. Retrieved January 15, 2014, from http://www.nintendolife.com/news/2013/01/goldeneye_creator_explains_n64_titl es_role_in_bringing_real_guns_to_the_virtual_world

Williams, D. (2006). Why game studies: Now gamers don't bowl alone. Games and Culture, 1(1), 13-16.

Willoughby, T., Adachi, P. J., \& Good, M. (2011). A longitudinal study of the association between violent video game play and aggression among adolescents. Developmental Psychology, 48(4), 1044-1057. doi:10.1037/a0026046

Wolf, M. J. P. (1997). Inventing space: Toward a taxonomy of on- and off-screen space in video games. Film Quarterly, 51(1), 11-23.

Wolf, M. J. P. (2012). Before the crash: Early video game history. Detroit, MI: Wayne State University Press.

Wolfenstein 3D. (1993). Tokyo, Japan: Imagineer. 
Woods, C., Serle, J., \& Ross, A. K. (2013). Emerging from the shadows: US covert drone strikes in 2012. The Bureau of Investigative Journalism. Retrieved January 15, 2014, from http://www.thebureauinvestigates.com/2013/01/03/emergingfrom-the-shadows-us-covert-drone-strikes-in-2012-2/

Zehnder, S. M., \& Lipscomb, S. (2004). Immersion in the virtual environment: The effect of a musical score on the video gaming experience. Journal of Psyiological Anthropology and Applied Human Sciences, 23(6), 337-343. 